\section{Intersections}

Canadian Journal of Music

Revue canadienne de musique
Intersections CANADIAN JOURAL OF MUSIC

\title{
La Musicologie du cinéma : enjeux disciplinaires et problèmes méthodologiques
}

\section{Chloé Huvet}

Volume 36, numéro 1, 2016

URI : https://id.erudit.org/iderudit/1043868ar

DOI : https://doi.org/10.7202/1043868ar

Aller au sommaire du numéro

\section{Éditeur(s)}

Canadian University Music Society / Société de musique des universités canadiennes

\section{ISSN}

1911-0146 (imprimé)

1918-512X (numérique)

Découvrir la revue

Citer cet article

Huvet, C. (2016). La Musicologie du cinéma : enjeux disciplinaires et problèmes méthodologiques. Intersections, 36(1), 53-84. https://doi.org/10.7202/1043868ar

\section{Résumé de l'article}

Si la musique de cinéma a longtemps été délaissée sur le plan scientifique, en particulier dans la francophonie, la floraison d'analyses musicologiques spécialisées dans ce domaine depuis quelques décennies commence toutefois à combler cette lacune. Par un regard réflexif porté sur ce jeune champ disciplinaire qu'est la " musicologie du cinéma », il s'agira d'interroger la place de l'étude de la musique de film au sein des travaux en musicologie, afin de comprendre le désaveu dont elle a pu faire l'objet. Bien que des disparités perdurent entre les musicologies nord-américaine et française, nous montrerons que l'institutionnalisation progressive de l'étude de la musique de film et son rattachement assumé à la musicologie semblent en bon chemin. L'exposé des problématiques propres à l'étude de la musique de cinéma permettra de dégager non seulement les défis méthodologiques posé par un tel objet de recherche, mais aussi d'éclairer la manière dont elles rejoignent et réengagent nombre de réflexions animant la discipline musicologique elle-même.
Copyright (C Canadian University Music Society / Société de musique des universités canadiennes, 2018
Ce document est protégé par la loi sur le droit d'auteur. L'utilisation des services d’Érudit (y compris la reproduction) est assujettie à sa politique d'utilisation que vous pouvez consulter en ligne.

https://apropos.erudit.org/fr/usagers/politique-dutilisation/ 


\title{
LA MUSICOLOGIE DU CINÉMA : ENJEUX DISCIPLINAIRES ET PROBLËMES MÉTHODOLOGIQUES
}

\author{
Chloé Huvet
}

En 1973, à l'occasion d'un discours prononcé à l'Eastman College de Rochester, le compositeur américain Bernard Herrmann déplorait:

L'utilisation de la musique dans les films est un domaine d'étude complètement délaissé. Autrefois, il existait des atlas mondiaux où les régions inexplorées étaient signalées en blanc et portaient l'étiquette "inconnu". Eh bien, il en va toujours de même pour la musique de cinéma ${ }^{1}$.

Herrmann trace ainsi l'écart paradoxal entre une pratique musicale quasiment incontournable au cinéma dès les premières projections à Paris en décembre 1895 et son investigation scientifique, qui a tardé à se construire. La musique de film est pourtant pour une grande part la continuation, transposée et transformée dans son application au cinéma, de conventions déjà fort anciennes et de toute une tradition de musique à programme ${ }^{2}$. Rappelons que le cinéma des premiers temps prend lui-même place dans les foires, les music-halls, les cafésconcerts et les spectacles de féerie ${ }^{3}$, la spécialisation des salles de projection ne se faisant que progressivement à partir de 19084. Associée à un art d'abord "populaire», la musique de film n’a pas été étudiée scientifiquement au même titre que les différents types de compositions faisant appel à une idée extra-musicale ou à un support verbal.

Depuis quelques décennies, la floraison d'études musicologiques spécialisées consacrées à la musique de film semble avoir répondu aux vœux d'Herrmann. En s'emparant de cet objet d'étude, les musicologues apportent une précision et une expertise non seulement dans l'analyse technique de la

1 Bernard Herrmann, cité dans Steven C. Smith, 2002, p. 360: "The use of music in films is a completely unstudied territory. In the old days there used to be atlases of the world with unexplored regions marked in white and labeled "unknown". Well, that's still what cinema music is like.» Toutes les traductions sont les nôtres.

2 Voir Chion 1995, p. 31-37.

3 La féérie est un spectacle qui, à la fin du XIX ${ }^{\mathrm{e}}$ siècle, repose sur les raffinements de la machinerie théâtrale et l'utilisation de l'électricité afin de produire des effets spectaculaires. (Voir Martin 2007).

4 Voir Barnier 2010. 
partition elle-même, mais aussi et surtout dans l'étude fine et la compréhension des relations entre la musique et les images, des transformations, des tensions et des reconfigurations mutuelles qui naissent de leur rencontre au sein du complexe audio-visuel.

Pourtant, cette vitalité exponentielle apparaît aussi comme un trompe-l'œil, donnant l'impression d'un élan homogène et généralisé qui masque un certain nombre de problèmes. Des disparités entre les musicologies nord-américaine et française dans la perception de la musique de cinéma comme objet d'analyse légitime perdurent. Dans le cadre circonscrit de cet article, nous avons choisi de nous concentrer principalement sur les recherches menées dans la sphère francophone, peu abordée dans des travaux similaires publiés en langue anglaise centrés sur les États-Unis et le Royaume-Uni5. Par ailleurs, les travaux historiographiques consacrés à la musique de cinéma par Martin Marks ou Robynn J. Stilwell consistent essentiellement en une recension (très exhaustive dans le cas de Stilwell) des principales publications consacrées à la musique de film depuis le cinéma muet jusqu’à nos jours.

Le présent article ne poursuit pas une telle visée - qui serait d'ailleurs quelque peu redondante vis-à-vis de ces travaux —, même si l'état de la recherche sera brièvement abordé. Par un regard réflexif porté sur ce jeune champ disciplinaire baptisé film musicology en anglais ${ }^{6}$, il s'agit ici d'interroger la place de l'étude de la musique de film au sein des travaux en musicologie, afin de comprendre le désaveu dont elle a longtemps fait l'objet7. L'exposé des problématiques propres à l'étude de la musique de cinéma nous permettra de dégager non seulement les défis méthodologiques auxquels les musicologues doivent faire face, mais aussi d'éclairer la manière dont elles rejoignent et réengagent nombre de réflexions animant la discipline musicologique elle-même.

Après un bref survol des premières recherches menées sur le sujet, nous verrons en quoi la musique de film a longtemps été un champ d'étude en quête de légitimité disciplinaire. Nous établirons ensuite de quelles manières une ouverture et les premiers signes de son institutionnalisation semblent se profiler - en particulier en France où, si l'ouverture est encore timide, le rattachement assumé de l'étude de la musique de film à la musicologie paraît toutefois en bon chemin. Enfin, nous explorerons les différents défis méthodologiques posés par un tel objet de recherche.

5 Voir Marks 1982, p. 3-40 ; Stilwell 2002, p. 19-61. Une recension historiographique a par ailleurs été établie par Pascal Pistone dans son article «Musicologie et cinéma. État des lieux, perspectives de recherche et bibliographie» (Pistone 2006, p. 89-129).

6 Voir Binns 2009, p. 725-738; et Rosar 2009, p. 99-125.

7 Nous adoptons dans notre texte une perspective œcuménique dans laquelle le terme de «musicologie» englobe une grande variété d'approches, notamment l'histoire de la musique, l'analyse, l'ethnomusicologie, l'étude des musiques populaires, etc. Ce caractère polymorphe des travaux scientifiques sur la musique est entériné entre autres par le choix du pluriel dans Jean-Jacques Nattiez 2003-2007, ou dans la collection «MusicologieS» chez Vrin. Nous emploierons donc les termes de «musicologie» et de "discipline» de manière englobante et au singulier par commodité, bien qu'ils doivent être entendus avec des guillemets. 


\section{LES PREMIÈRES ÉTUDES SUR LA MUSIQUE DE CINÉMA}

\subsection{Une impulsion initiée aux États-Unis}

Il peut paraître surprenant que l'étude de la musique de film se soit développée en marge à la fois de la musicologie et des études cinématographiques. Des écrits consacrés à cet objet sont certes publiés dès les années 1910, mais il ne s'agit pas d'études scientifiques. Dans une visée pratique, ces ouvrages sont écrits par des musiciens à l'attention des accompagnateurs de films muets. Des recueils appelés "catalogues d'incidentaux ${ }^{8}$ » donnent des indications de tempo, de caractère et suggèrent des extraits de partitions les mieux appropriés pour chaque situation dramatique ${ }^{9}$.

En dehors de cette première littérature, on ne peut qu'être frappé par le silence qui enveloppe nombre d'écrits filmiques, dénoncé relativement tôt par des chercheurs en cinéma eux-mêmes, au premier rang desquels Claudia Gorbman $^{10}$. Les premières études menées sur le sujet ont été conduites aux ÉtatsUnis. L'ouvrage de Claudia Gorbman publié en 1987 apparaît ainsi comme une véritable pierre fondatrice pour l'étude de la musique de cinéma, dont les répercussions sont immédiates dans le monde anglo-saxon ${ }^{11}$. Appliquant une méthodologie inspirée de la sémiologie, de la narratologie, du structuralisme et des théories de la psychologie musicale qu'elle croise avec l'analyse filmique, Gorbman ambitionne de répondre à deux questions principales: pourquoi, dès le cinéma muet, les films ont-ils eu besoin d'une présence musicale, et de quelles manières la musique agit-elle sur la perception du spectateur? En définissant plusieurs concepts clés - en particulier la distinction entre musique diégétique et extra-diégétique issue de la narratologie - et en posant un cadre conceptuel clair autour des principales fonctions de la musique au cinéma, Gorbman jette ainsi les bases théoriques et fournit les premiers outils d'une approche rigoureuse de la musique de film. Les trois études de cas qui ferment le livre - traitement de la partition de Maurice Jaubert pour Zéro de conduite (1933), travail sur la musique et l'espace sonore dans Sous les toits de Paris (1930), et caractère "anempathique» de la musique de Bernard Herrmann dans Hangover Square (1945) - offrent par ailleurs des modèles analytiques diversifiés, qui ont été repris, adaptés et élargis par la suite par d'autres chercheurs. Le travail de Gorbman apparaît en effet comme le véritable catalyseur d'une série d'études scientifiques en langue anglaise sur le sujet. Concentrés entre 1992 et 1995, ces ouvrages sont les premiers à tenter une théorisation de la musique de film, doublée d'une approche à la fois analytique à travers des études de cas ponctuelles, mais aussi historique et esthétique, dégageant les grands traits stylistiques de quelques courants majeurs à la manière de ce qu'avait entrepris Gorbman pour le symphonisme hollywoodien ${ }^{12}$. Les recherches anglophones

8 Parmi les plus célèbres, citons Becce 1920-1927; Rapée 1925.

9 Voir Marks 1997, p. 3-61 ; Pisano 2002.

10 Gorbman 1987 ; Gorbman 1995, p. 72-75.

11 Gorbman 1987.

12 Voir Kalinak 1992 ; Flinn 1992 ; Brown 1994. 
en musicologie du cinéma connaissent depuis une vitalité remarquable, que nous aborderons plus loin.

\subsection{Un objet d'étude abordé tardivement en France}

Pour sa part, comme le rappelle Jérôme Rossi, la France «ne peut se prévaloir d'une telle tradition dans la recherche en musique de film ${ }^{13}$ ». Peut-être en raison du poids plus prégnant exercé par le discours des avant-gardes, la littérature française en musicologie du cinéma s'est développée plus tardivement. Certes, quelques propositions sont contemporaines des premiers écrits anglo-saxons ${ }^{14}$, mais contrairement aux livres de Gorbman ou Kalinak qui demeurent des ouvrages de référence incontournables, ces approches paraissent datées voire trop lâches ${ }^{15}$. Les ouvrages de Michel Chion publiés dans les années 1990 constituent une exception notable, faisant autorité aussi bien dans les recherches francophones qu'anglophones, grâce à la terminologie et à un ensemble d'outils essentiels à l'analyse audio-visuelle qui y sont développés ${ }^{16}$. Chion étend les concepts et méthodes de Gorbman, tout en proposant de nouvelles notions comme la valeur ajoutée évoquée plus haut, l'anempathie ou l'acousmêtre, pour n'en citer que deux ${ }^{17}$.

\section{UN CHAMP D'ÉTUDE EN QUÊTE DE LÉGITIMITÉ DISCIPLINAIRE. RAISONS D'UN DÉSAVEU ORIGINEL}

Comment comprendre un essor si tardif des études scientifiques sur la musique de cinéma? L'ostracisme dont elle a longtemps fait l'objet résulte d'un entrelacement complexe de présupposés historiques, idéologiques et esthétiques dont il convient de démêler les fils.

\subsection{La sourde oreille des études cinématographiques}

Du côté des chercheurs en cinéma, plusieurs auteurs comme Caryl Flinn, William Darby, Jack Du Bois ou Anahid Kassabian, possédant pourtant des bases musicales, témoignent tout d'abord d'une méfiance vis-à-vis du vocabulaire musical technique et des exemples sur portées, jugés rédhibitoires pour les lecteurs non spécialisés ${ }^{18}$. Gorbman recourt certes à des exemples musicaux

13 Rossi 2016, p. 20.

14 Voir en particulier Boilès 1975, p. 71-85; et Julien 1980, p. 179-202.

15 C'est le cas de Julien, qui projette d'«établir une typologie qui distingue un certain nombre de situations dans lesquelles le compositeur [...] installe une musique pour des images» (Julien 1980, p.180). Les catégories analytiques qu'il dégage ensuite sont assez confuses et se chevauchent les unes les autres. Citons par exemple «la musique dans ses lieux» (p. 182), «les musiques de parcours» (p. 186), «les musiques en situation psychologique» (p. 189), «les musiques de loisirs» (p. 195) ou «les musiques du regard» (p. 196).

16 Chion 1990 et 1995.

17 Un effet anempathique est créé quand «la musique, lors d'une scène particulièrement éprouvante (meurtre, torture, viol, etc.), affiche son indifférence en continuant son cours comme si de rien n'était» (Chion 1995, p. 229). Un acousmêtre est un être entièrement acoustique, qui existe sous forme de voix ou de son désincarné sans que sa source physique ne soit montrée à l'écran, ce qui lui confère un fort potentiel dramatique. (Voir Chion 1990, p. 109-10).

18 Voir Flinn 1992, p. 12 ; Kassabian 2001, p. 9. 
d'une ou deux mesures sur une seule portée, mais certains sont renvoyés en note de bas de page à la fin de l'ouvrage, ce qui pose la question de la réelle importance de ces transcriptions aux yeux de l'auteur comme pour le lecteur. Darby et Du Bois optent eux aussi pour une présentation extrêmement simplifiée, qui s'avère particulièrement problématique par exemple pour le thème principal "Ave Satani» de La Malédiction (1976). En effet, les auteurs tentent de démontrer l'originalité de la composition de Jerry Goldsmith (1929-2004), à laquelle la seule transcription mélodique proposée - un si répété sur quatre mesures - ne rend guère justice ${ }^{19}$. En effet, la spécificité de ce $c u e^{20}$ réside dans le travail réalisé sur le timbre et le langage harmonique à travers le mélange de stridences dissonantes de cordes et de cuivres, les ponctuations de timbales et les incantations du chœur mixte présentant de fréquents glissements chromatiques tendus.

Par ailleurs, la primauté longtemps accordée au visuel sur le sonore par des théoriciens influents comme Béla Balázs et Rudolf Arnheim relègue la musique et les bruitages à une place secondaire dans les écrits sur le cinéma, comme si le son était uniquement pensé et ajouté à l'image après coup ${ }^{21}$. Que l'accompagnement musical prenne la forme d'un renforcement ou d'une opposition, cela présuppose que le sens est déjà là, contenu dans l'image. Les relations musique/ image ont ainsi longtemps été abordées à travers le concept binaire synchronisme/contrepoint: la musique aurait soit la possibilité de venir appuyer ce qui est entièrement contenu dans l'image et la narration, soit en altérerait le sens par une apparente contradiction ${ }^{22}$. Serge Cardinal rappelle à juste titre:

[Dans cette conception], la musique n’a plus pour rôle général que de réagir à ce qui est posé ou donné. C’est à croire que les cinéastes [...] ne sont que de mauvais artistes peintres, commençant par dessiner leurs figures avant de les remplir de couleurs, et le spectateur, un drôle d'amateur de peinture qui séparerait le dessin de la couleur sous prétexte que l'un a été tracé avant que l'autre ne soit appliquée ${ }^{23} \ldots$

En réalité, un mécanisme inconscient s’opère, théorisé par Michel Chion sous l'expression de "valeur ajoutée», cette "valeur sensorielle, informative, sémantique, narrative, structurelle ou expressive qu'un son entendu dans une scène nous amène à projeter sur l'image, jusqu'à créer l'impression que nous voyons dans celle-ci ce qu'en réalité nous y "audio-voyons" $24 »$. Le processus fonctionne d'ailleurs à double sens: la musique est elle-même affectée, réévaluée par le visuel. L’image la fait entendre d'une autre manière, selon le contexte. Chion prend comme exemple le son d'une pastèque s'écrasant au sol. Le contexte lui donnera des significations fort différentes: dans une comédie,

19 Darby et Du Bois199o, p. 507.

20 Un cue désigne chaque musique entendue à partir de son signal d'entrée jusqu'à son interruption au sein du film.

21 Voir en particulier Carter 2010 ; Rick Altman dans son introduction «Four and a Half Film Fallacies» (Altman 1992, p. 35-45).

22 Voir Kracauer 1960, p. 139-146; Gorbman 1980, p. 183-203.

23 Cardinal 2012, p. 46.

24 Chion 2010, p. 162. 
il prêtera à rire, tandis que dans un film d'horreur il pourra susciter frisson et dégoût, comme l'a remarquablement mis en scène le réalisateur britannique Peter Strickland dans Berberian Sound Studio (2012). Dans cette perspective, Nicholas Cook cite quant à lui un exemple musical: le thème accompagnant la fuite de Marion Crane sous la pluie battante avant son arrivée au Bates Motel au début de Psychose (1960) n'illustre pas ce qui se déroule à l'image. Par son contour anguleux, ses répétitions obsédantes et son instrumentation réduite formée uniquement de cordes, la musique incarne plutôt le malaise croissant du personnage, creusant l'image afin de mettre au jour le trouble intérieur de Marion, tandis que, d'un autre côté, le contexte visuel confère à l'accompagnement une dimension sinistre qu'il ne possède pas en lui-même ${ }^{25}$.

Rappelons ici une évidence: ne pas tenir compte de la musique dans une analyse de séquence conduit facilement à des interprétations erronées ${ }^{26}$. Des chercheurs anglo-saxons comme Kathryn Kalinak, Amy McGill et Jay Beck, ou francophones tels Serge Cardinal, Martin Barnier, Laurent Guido et Pierre Berthomieu, démontrent au contraire tout l'intérêt que peuvent trouver les études cinématographiques à tendre une oreille attentive à la musique ${ }^{27}$.

\subsection{Un objet d'étude indigne de la musicologie?}

Du côté de la musicologie, loin des canons la culture musicale, la musique de film a longtemps pris la forme d'une terra incognita voire d'un objet d'étude peu recommandable ${ }^{28}$. Tenter de comprendre les raisons d'un tel opprobre apporte en filigrane plusieurs éléments d'éclairage sur la discipline elle-même.

La fonctionnalité de la musique de cinéma, sa subordination à des éléments qui lui sont extérieurs et le fait qu'elle ne soit pas conçue pour être entendue et jouée en concert, forme qui s'est progressivement cristallisée comme le modèle de l'écoute savante dans les sociétés occidentales ${ }^{29}$ et qui atteste en creux la qualité des œuvres jouées, expliquent sa relative mésestime ${ }^{30}$. Ce type de considérations idéologiques s'inscrivent en effet dans la continuité de débats déjà fort anciens autour de la supériorité alléguée de la musique "pure», qui ont irrigué l'histoire de la musique à programme et sont loin d'être clos $^{31}$.

Le conditionnement de la logique interne de la partition par le film comporte d'autres implications importantes. Dans la mesure où le matériau tire sa logique et son organisation du film, les grandes formes de la musique savante occidentale sont majoritairement absentes au cinéma, ou y sont utilisées

25 Chion 1998, p. 66-67.

26 Voir Larsen [2005] 2007, p. 110-22.

27 Voir Kalinak 2007; McGill 2008; JBeck. 2013, p. 732-51; Cardinal, Allard et Comtois 2002, p. 158-74; Barnier 2007, p. 41-49; Guido 2006, p. 52-75; Berthomieu 2011.

28 Alain Poirier dénonce et réfute ces jugements dépréciatifs au début de son article «Les fonctions de la musique au cinéma» (dans Nattiez 2003, p. 750-55).

29 Voir Bödeker, Veit et Werner 2006; Martin Kaltenecker 2010; Sarah Barbedette 2014.

30 D'autres traditions musicales, dont le jazz (bien qu'intégré relativement tôt en musicologie), la musique populaire ou la musique de jeu en ont également souffert dans un premier temps.

31 Voir en particulier Hanslick [1854] 2012; Rodgers 2009). Sur les considérations autour de la notion de «musique pure», voir Levinson 1990, p. 90-125; Cook 1998, p. 86-97 et p. 265-272. 
dans un esprit très libre ${ }^{32}$. Les techniques traditionnelles d'analyse formelle, qui constituent un pan essentiel de l'approche musicologique ${ }^{33}$, se trouvent ainsi relativement démunies face à l'invention nouvelle dont font montre les structures musicales cinématographiques. D’autant que la musique au cinéma présente la particularité d'être intermittente, revêtant un aspect fragmenté et éclaté à l'échelle du film.

Le maintien d'un langage harmonique en grande partie tonal dans la plupart des partitions de cinéma des $\mathrm{XX}^{\mathrm{e}}$ et $\mathrm{XXI}^{\mathrm{e}}$ siècles constitue un autre nœud de leur réception dépréciative en musicologie, dans la mesure où il apparaît en décalage patent vis-à-vis de la production savante depuis l'émergence de la seconde école de Vienne. Il ne faut pourtant pas oublier que la conservation d'un langage hérité du Romantisme et du post-Romantisme découle d'abord de conventions du cinéma muet, où les «catalogues d'incidentaux» regroupaient des partitions célèbres de musique classique et populaire principalement issues des XVIII et $\mathrm{XIX}^{\mathrm{e}}$ siècles, destinées à une utilisation dramatique spécifique ${ }^{34}$. L’origine et la formation des compositeurs majeurs qui ont façonné le modèle influent du symphonisme hollywoodien dès les années 1930 jouent aussi un rôle non négligeable dans la perpétuation de l'idiome tonal, de types d'écriture et d'orchestration hérités du XIX ${ }^{\mathrm{e}}$ siècle: la plupart d'entre eux, nés en Europe de l'Est, émigrent aux États-Unis à la fin des années 1920 à la suite de la montée de l'antisémitisme, tels Max Steiner (1888-1971), ancien élève de Gustav Mahler, ou Erich W. Korngold (1897-1957), musicien à l'opéra de la cour de Vienne.

Par son intégration modérée des techniques d'écriture et du langage de la musique contemporaine savante, la musique au cinéma est souvent jugée conservatrice et de peu d'intérêt, en vertu du «système de valeurs [...] en place dans notre culture [qui] fait passer l'innovation avant la tradition, la création avant la reproduction, l'expression personnelle avant le marché 35 ». Dans leur essai de 1944, qui compte parmi les premiers ouvrages majeurs sur la musique de cinéma, Theodor W. Adorno et Hanns Eisler fustigent ainsi le recours majoritaire à un «langage conventionnel préfabriquéz ${ }^{6}$ » hérité de la musique romantique véhiculant selon eux des références préétablies et figées, et l'usage du leitmotiv, réduit au cinéma à un "valet de chambre musical 37 ». La condamnation du modèle hollywoodien conduit Adorno et Eisler à valoriser l'utilisation de la musique «moderne»: dans la mesure où elle permet d'éviter l'écueil de la redondance et de l'illustration musicales dominant à leurs yeux le cinéma

32 C'est le cas du fugato et de la passacaille, que l'on peut rencontrer au cinéma à l'occasion. Pour les fugatos, voir le générique d'ouverture composé par Jacques Demy pour Peau d'âne (1970) et «The Shark Cage Fugue» écrit par John Williams pour Les dents de la mer (1975). Des passacailles «libres» interviennent juste avant le meurtre de Leona Stevenson dans la partition de Franz Waxman pour Raccrochez, c'est une erreur (1948), l'invasion du Tibet par la Chine dans la musique de Philip Glass pour Kundun (1997). Pour davantage de précisions, voir Brown 1994, p. 47-48, et Riesman 2016, p. xiii.

33 Voir Adorno[1982] 2002, p. 162-180; Donin 2009, p. 22-27.

34 Voir Mouëllic 2003, p. 6-7.

35 Cook [1998] 2006, p. 23. Nous reviendrons plus loin sur cette question de la valeur commerciale.

36 Adorno et Eisler [1944] 1972, p. 42.

37 Ibid., p. 15. 
commercial, elle semble être seule en mesure de susciter de nouvelles relations entre la musique et les images. Dans la littérature, les emprunts aux techniques d'écriture de l'avant-garde musicale tendent, de fait, à être associés de manière exclusive au cinéma d'auteur ou indépendant, la «nouveauté» ou le caractère expérimental de la partition servant souvent à démontrer la qualité d'«auteur» du réalisateur et sa distinction vis-à-vis des productions hollywoodiennes ${ }^{38}$, oubliant que les procédés d'écriture de la musique contemporaine sont intégrées dans nombreuses partitions filmiques du cinéma mainstream ${ }^{39}$. Du côté des metteurs en scène, ce recours à un langage dissonant généralisé a souvent pris la forme d'un quasi manifeste, d'une réaction vis-à-vis d'un modèle esthétique dominant et des conventions passées, en particulier dans le cinéma français de la Nouvelle Vague ${ }^{40}$; par sa nature et la manière dont il s'articule avec l'image, ce type de langage éloignerait l'accompagnement musical du rôle descriptif et des "associations automatisées ${ }^{41}$ » que dénonçaient Adorno et Eisler. Il convient toutefois de souligner l'éclectisme propre à la musique de cinéma qui, loin de se limiter au langage post-romantique, intègre des influences très diverses, des musiques non-occidentales, du folklore, du minimalisme et de l'atonalité aux chansons pop-rock ou au jazz ${ }^{42}$.

\subsection{Musique de film et statut de la création artistique}

Outre ces aspects intrinsèques à la musique de film, plusieurs dimensions externes achèvent d'expliquer la déconsidération dont elle a longtemps fait l'objet dans le milieu musical, parce qu'elles interrogent la notion même de création et son statut.

Le matériau musical tel qu'entendu au montage final d'un film résulte d'un processus de création collectif, au-delà des choix du compositeur seul. Au moment de l'écriture, ce dernier doit non seulement prendre en considération la présence des autres matériaux sonores et les indications du metteur en scène et des producteurs, mais surtout s'accommoder des décisions prises en post-production. Des sections voire des cues entiers sont fréquemment tronqués, rallongés artificiellement, déplacés à d'autres endroits du film, soit parce qu'ils fonctionnent mieux avec une autre scène, soit pour répondre à des changements apportés au montage image, bien souvent sans tenir compte de leur structuration originelle ni consulter le compositeur. Au mixage, selon les

38 Voir en particulier Kulezic-Wilson 2008, p. 127-128 et Kickasola 2012, p. 61-75.

39 Pour ne citer que quelques exemples, John Williams recourt à l'atonalité et à l'athématisme dans La guerre des mondes (2005) où les interventions musicales sont par ailleurs brèves et fragmentées, tandis que les «amalgames soniques» qui jalonnent Inception (2010) ou Mad Max: Fury Road (2015) orientent la bande sonore dans son ensemble vers une forme singulière de musique électroacoustique, déplaçant les frontières habituellement établies entre musique et sound design. Voir Rodman 200o, p. 187-206; Rossi 2011, p. 113-40; Wright 2015, p. 319-27.

40 Pour Eric Rohmer (Sous le signe du lion, 1959, musique de Louis Saguer), Alain Cavalier (Le combat dans l'île, 1961, musique de Serge Nigg) ou Claude Chabrol (Le boucher, 1970, musique de Pierre Jansen), la musique de l'avant-garde peut répondre aux articulations formelles plus souples et à la plus grande liberté du montage. Voir Gimello-Mesplomb 2016, p. 109-32.

41 Adorno et Eisler [1944] 1972, p. 44.

42 Voir M. Eaton 2008; Minett 2013, p. 191-210; Mouëllic 2000. 
besoins des séquences, le volume de l'accompagnement orchestral est artificiellement ajusté, ce qui peut conduire à la quasi inaudibilité des phrases musicales, qu'elles soient ou non névralgiques du point de vue de l'organisation interne du discours.

Par ailleurs, le fait que le compositeur de cinéma n'œuvre pas seul pose la question de la paternité réelle de l'accompagnement musical: des orchestrateurs, co-compositeurs ou arrangeurs complètent ses esquisses afin de respecter les délais d'écriture relativement courts - quatre à six semaines en moyenne ${ }^{43}$. Ce phénomène a pris une ampleur nouvelle à l'ère numérique; le rôle de premier plan joué sur la scène hollywoodienne par le studio Remote Control Productions dirigé par Hans Zimmer en est peut-être la manifestation la plus extrême. Pirates des Caraïbes: la malédiction du Black Pearl (2003) offre un aperçu exemplaire de ce processus de création. La partition d'Alan Silvestri ayant été rejetée, le projet est confié à Klaus Badelt qui dispose de trois semaines pour composer plus de 90 minutes de musique: sous la supervision de Zimmer, il fait alors appel à une quinzaine d'orchestrateurs, co-compositeurs et arrangeurs, et de nombreux cues sont écrits à plusieurs mains ${ }^{44}$.

Pour faire face aux impératifs d'efficacité et de rentabilité dans le temps alloué, les compositeurs usent par ailleurs de ficelles, de codifications musicales qui fonctionnent parfaitement pour tel type de situation dramatique ou font partie des attendus génériques ${ }^{45}$. Engagés en regard de leur production passée, ils recyclent également certaines de leurs partitions à la demande des réalisateurs et des producteurs qui misent sur un succès antérieur.

Enfin, les considérations financières fondamentales et la position «subalterne» de la musique par rapport à l'image tendent à jeter un discrédit sur sa qualité. Alors que des critiques musicaux comme Émile Vuillermoz ou Jean d'Udine louent les possibilités nouvelles que leur semble receler la musique de cinéma et que plusieurs compositeurs de la tradition savante se tournent vers le médium cinématographique ${ }^{46}$, l'ensemble des mouvements d'avant-garde, dont le discours particulièrement influent laissera une empreinte, renie la musique de film avec véhémence ${ }^{47}$. À la suite de son expérience décevante sur la Symphonie mécanique (1955), Pierre Boulez déplore l'implication du compositeur à un stade tardif de la production cinématographique, qui le réduit à n'être qu'un «boucheur de trous ${ }^{48}$ ». Les jugements dépréciatifs portés par les compositeurs savants eux-mêmes ont ainsi participé à décrédibiliser la musique de

43 Kalinak 2010, p. 94. Voir aussi Karlin et Wright [1990] 2004, p. 60.

44 Pour davantage de détails sur le mode de fonctionnement de Remote Control, voir Wright 2015, p. 319-327; Huvet 2015, p. 119-22.

45 Voir Kalinak 1982, p.76-82; Neumeyer et Buhler 2001, p. 23-26.

46 Voir Poirier 2003, p. 760-64; Gonin 2008; Descheneaux 2010, p. 475-91; Chanudaud 2016, p. 35-52; Zwein-Chouard 2016, p. 97-107.

47 Voir Oddos et Carcassonne 1979, p. 27; Calabretto 2002. Évoquant l'avant-garde italienne, l'auteur écrit: "Caractéristique est à cet égard l'attitude de la "génération des années quatre-vingt" italienne et ses membres, avant tout Malipiero et Pizzetti, qui renièrent les films auxquels ils avaient collaboré» (note 9).

48 Oddos et Carcassonne 1979, p. 27. Cette appellation est une constante de nombreux écrits français sur le cinéma sonore. Voir Jaubert 1936, p. 115; Hacquard 1959, p. 66; Langlois 1968, p. 22. 
cinéma par rapport à la production savante. Francis Poulenc affirme que «la musique de cinéma reste une tâche inférieure et vénale ${ }^{49}$ », tandis que selon Igor Stravinsky, «le seul intérêt de la musique de film est de nourrir son compositeur ${ }^{50}$ ». Cette dévalorisation est vécue comme un fort rejet par les compositeurs ayant choisi de se spécialiser dans le cinéma, tel Georges Delerue ${ }^{51}$.

La réalité propre à la musique de cinéma pose plusieurs problèmes dans une conception musicologique traditionnelle. La réflexion autour du réemploi, du recyclage de sources et de matériau passés comme supports à de nouvelles créations irrigue certes depuis longtemps l'histoire des arts, où l'invention se joue dans un processus de migration, de citations ou de détournements. Mais ce recours à l'emprunt relativement commun dans l'histoire de la musique savante, qui est au fondement de l'esthétique dite "postmoderne ${ }^{52}$ et dont la légitimité semble admise dans la musique contemporaine où il peut prendre la valeur d'une "activité résolument moderniste ${ }^{53}$ ", est interprété au cinéma comme le signe d'un manque d'inventivité parce qu'il est compris comme une facilité dictée par une logique de rentabilité54. Par ailleurs, l'entremêlement des rôles au sein du département musique rend très difficile de discerner la responsabilité réelle de chacun des collaborateurs. De telles pratiques contredisent non seulement l'idéal d'organicité, et de cohérence interne à l'œuvre issu de l'esthétique romantique qui a façonné une partie de la musicologie, mais mettent également en crise la conception traditionnelle et idéalisée du compositeur comme instigateur et responsable de la totalité du matériau musical55. Les questions posées par la musique de film rejoignent ainsi des remises en cause animant la musicologie actuelle ${ }^{56}$.

49 Francis Poulenc, cité dans Machart 1995, p. 75.

50 Stravinsky 1947, p. 3.

51 Le compositeur regrette: "Cette étiquette est gênante parce que les musiciens "sérieux" ont tendance à considérer les musiciens de film comme des parias faisant partie du show business. On est suspect parce qu'on gagne de l'argent!» (Cité dans Rebichon 1987, p. 96).

52 Voir en particulier Ramaut-Chevassus 1998 ; Gloag 2012.

53 Goldman 2008, p. 8. Voir aussi les travaux de J. Peter Burkholder se penchant sur les stratégies d'emprunts dans la musique de Charles Yves, notamment son ouvrage All Made of Tunes: Charles Ives and the Uses of (Burkholder 1995).

54 À cet égard, nul n’a autant été stigmatisé que James Horner (1953-2015). Lors du colloque Music \& the Moving Image organisé à l'Université de New York en mai 2016, pas moins de deux communications étaient exclusivement consacrées à la question de «l'auto-plagiat» dans les partitions de Horner (Michael W. Harris, «Borrowing Beyond the Stars: James Horner's Music for Star Trek II and III»; Nicholas Kmet, «The Danger Theme: The Question of Self-Plagiarism and Recycling Musical Material in Film Music»). Hans Zimmer et John Williams sont eux aussi les cibles d'accusations de plagiat et d'auto-emprunts peu subtils (voir Orosz 2015, p. 299-319).

55 Ces questionnements font écho dans le domaine des arts aux recherches menées sur l'atelier des peintres, et aux polémiques littéraires autour des ghostwriters, ces plumes anonymes tenues secrètes. La notion d'auteur est mise en crise depuis longtemps dans les études littéraires, notamment par le célèbre article de Barthes intitulé «La mort de l'auteur». Selon Barthes, l'auteur comme principe producteur de l'œuvre disparaît derrière le langage même, aux mots: «L'écriture, c'est ce neutre, ce composite, cet oblique où fuit notre sujet, le noir-et-blanc où vient se perdre toute identité, à commencer par celle-là même du corps du corps qui écrit» (Barthes 1984, p. 61).

56 Voir notamment Soury 2013, p. 33-60. Dans le domaine du jazz et des musiques populaires, le rôle de l'arrangeur, de l'interprète ou de l'enregistrement vis-à-vis du compositeur a été récemment interrogé. Voir en particulier Cugny 2009 et Lacasse 2006, p. 65-78. 


\section{VERS UNE INSTITUTIONNALISATION DE LA MUSICOLOGIE DU CINÉMA?}

\subsection{Ouvertures (intra)disciplinaires}

Dans ces conditions, un canon s'est mis en place dès les années 1980, délimitant les partitions méritant d'être analysées à travers un nombre choisi de compositeurs jugés légitimes. Les premiers chercheurs anglo-saxons se sont intéressés aux mêmes musiciens pionniers du symphonisme hollywoodien - en particulier Steiner et Korngold, dont la formation permettait indirectement de faire rejaillir le prestige attaché à la musique savante sur le modèle classique américain, justifiant du même coup son investigation scientifique 57 . La figure de Bernard Herrmann constitue un deuxième volet majeur du canon qui découle d'un postulat auteuriste: la majorité des auteurs s'intéressent à sa collaboration avec Alfred Hitchcock, délaissant son travail jugé moins prestigieux pour les films fantastiques (L'île mystérieuse, 1961) ou les péplums tels Le $7^{e}$ Voyage de

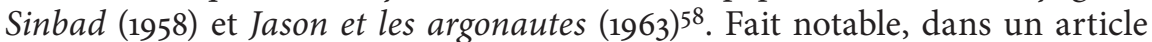
au titre éloquent, William $\mathrm{H}$. Rosar inscrit la littérature herrmannienne dans le sillon du canon beethovenien ayant longtemps imprégné la musicologie 59 . Dernier élément constitutif du canon, le cinéma d'auteur français se dédouble entre le modèle esthétique érigé par Maurice Jaubert en réaction contre le symphonisme hollywoodien ${ }^{60}$ et les pratiques «modernistes» de la Nouvelle Vague $^{61}$. Dans la littérature imprégnée de l'idéal jaubertien se joue en filigrane l'affirmation de l'existence et de la pérennité d'une musique spécifiquement française, recoupant des questionnements musicologiques qui se poursuivent encore de nos jours ${ }^{62}$.

Depuis la fin des années 2000, toutefois, le paysage de la musicologie du cinéma connaît une mutation indéniable. La progressive sortie du canon se traduit par la légitimation scientifique d'objets d'étude autrefois délaissés, au premier rang desquels les blockbusters, ces films péchant doublement par «les sommes colossales investies dans la production et le marketing, ainsi que le montant des revenus reçus» qui les définissent ${ }^{63}$. Les chercheurs se sont ainsi emparés des sagas populaires Harry Potter, Superman, Jason Bourne, Jurassic

57 Signe de la persistance de ce canon, la majorité des Film Score Guides, collection spécialisée proposant des études approfondies d'une partition, sont dédiés aux compositeurs phares du classicisme américain. Citons Daubney 2000; Winters 2007; Hickman 2011; Neumeyer et Platte 2011.

58 La somme des écrits sur Herrmann est parmi les plus conséquentes en musique de film. Nous ne citons ici que les principaux: Brown 1982, p. 14-49; Bruce 1985; Smith 2002 ; Eugène 2000; Cooper 2001 et 2005; Carayol 2015, p. 173-85.

59 Rosar 2003, p. 121-50. Voir Kerman 1983, p. 107-25; DeNora 1998.

60 Voir Porcile 1971); Lacombe et Porcile 1995.

61 Lacombe et Porcile affirment avec emphase qu’avec la Nouvelle Vague, «le cinéma n'est plus pour le musicien l'antre maudit où l'on gagne sa vie et perd son âme» (Lacombe et Porcile 1995, p. 274). La figure de Jean-Luc Godard en particulier occupe une place prépondérante dans la littérature. Voir Brown 1994, p. 188-234; Jullier 2009, p. 352-62; Roustom 2014, p. 71-88.

62 Voir Terrien 2014; Reibel 2015, p. 73-84.

63 Buckland 2006, p. 17: «[A blockbuster can be defined in terms of two variables] : the huge sums involved in production and marketing, and the amount of revenues received.» Sur la déconsidération envers les blockbusters, voir Jullier 2011, p. 189-91. 
Park ou Batman ${ }^{64}$. D’autres genres jadis jugés moins nobles que les productions cinématographiques, comme les séries télévisées ${ }^{65}$ ou les jeux vidéo ${ }^{66}$, ont fait l'objet d'analyses musicologiques récentes, tandis qu'un projet d'envergure inédite est mené depuis 2012 autour du son des bandes-annonces ${ }^{67}$. Cette déconcentration s'inscrit ainsi pleinement dans l'évolution de la notion de valeur artistique dans les sociétés contemporaines, qui a pour corollaires l'abandon progressif d'un répertoire phare ainsi que le renouvellement des notions considérées culturellement et institutionnellement légitimes ${ }^{68}$.

\subsection{Cadre académique et diffusion de la recherche}

Il est intéressant de constater que cette extension des objets d'étude au sein de la musicologie du cinéma reflète l'ouverture, à plus large échelle, de la discipline musicologique elle-même à un répertoire longtemps réputé moins prestigieux - comme ce fut le cas précédemment avec le jazz, ou la musique populaire. À cet égard, il convient de distinguer les musicologies anglo-saxonne et française. La musicologie du cinéma est mieux implantée aux États-Unis et au Canada. Elle est intégrée depuis longtemps aux parcours universitaires de nombreux établissements américains comme l'Université de Californie à Los Angeles, l'Université de Californie du Sud, l'Université de New York, où l'enseignement est divisé entre composition et cours théoriques ${ }^{69}$. Le Carleton College du Minnesota, l'Université de Caroline du Sud et l'Université du Texas à Austin proposent une approche théorique et historique de l'évolution de la musique de film. Au Canada, les cours donnés à l'Université du Québec à Montréal depuis 2007 et à l'Université Ryerson ont une visée professionnalisante, tandis que l'enseignement proposé à l'Université Carleton et à l'Université de Montréal depuis 2002 est dédié à l'étude des théories, des techniques et des styles, à l'analyse et à l'histoire de la musique de cinéma, et aux poétiques de l'audio-visuel, dans une visée interdisciplinaire. Au sein de l'Université de Montréal, le laboratoire de recherche-création «La création sonore: cinéma, arts médiatiques, arts du son» se distingue particulièrement par son dynamisme, la diversité des recherches qui y sont menées, et l'organisation de nombreuses classes de maitre.

En France, la majorité des enseignements, tournés vers la pratique et la professionnalisation, sont destinés aux étudiants compositeurs, tels le Master

64 Webster 2009; Halfyard 2013, p. 171-93; Buhler et Newton 2013, p. 325-49; Huvet 2014, p. 2339 et Hexel 2016.

65 Stilwell 2011, p. 119-42; Michot 2012; Carayol et Rossi 2015.

66 Collins 2008; Donnelly, Gibbons et Lerner 2014; Doucet 2016. Un ouvrage récent nomme la branche disciplinaire: Kamp, Summers et Sweeney 2016.

67 Le projet Trailaurality autour de la musique et du sound design des bandes-annonces, financé par le Centre de Recherches en Sciences Humaines du Canada, est conduit à l'Université Carleton par James Deaville, Dawn Stevenson, Curtis Perry et Jordan Zalis. Des résultats partiels de leurs recherches sont publiés sur http://www.trailaurality.com/. Voir aussi Deaville et Malkinson 2014, p. 121-40.

68 Voir notamment les travaux des sociologues Peterson 1992, p. 243-58 et Lahire 2004.

69 Signe de l'ancienneté de l'implantation de ces formations universitaires, le compositeur Miklós Rózsa (1907-1995) a par exemple enseigné la composition musicale pour le cinéma à l'Université de Californie du Sud dès 1945. 
«Musiques Appliquées aux Arts Visuels» créé à l'Université Lumière Lyon 2 en 2007, le cursus "musique à l'image» organisé en trois cycles au Conservatoire National Supérieur de Musique et de Danse de Lyon depuis 2004, et la préparation d'un an au "Diplôme de composition de musique à l'image» du CNSMDP (Conservatoire National Supérieur de Musique et de Danse de Paris), ouvert début 2013. Des cours théoriques et des séminaires de recherche existent, notamment dans les universités Paris-Sorbonne, Rennes 2 ou depuis 2013 à l'Université de Rouen, mais ils ne bénéficient pas de la même visibilité et restent assez isolés au sein du cursus musicologique français.

Sur le plan de la diffusion académique de la recherche aux États-Unis, le type d'approches évoquées précédemment et initiées par Gorbman et Kalinak cèdent le pas à des études de cas approfondies qui font l'objet d'ouvrages entiers (souvent publiés dans la collection Film Score Guides), et à des analyses musicales centrées autour des genres cinématographiques - en particulier l'horreur, la pornographie, le fantastique et la comédie ${ }^{70}$. Les chercheurs américains se sont également saisis des aspects techniques de la production musicale filmique, intégrant récemment les notions de montage, de post-production numérique et de sound design aux considérations musicales traditionnelles ${ }^{71}$. Le colloque transdisciplinaire annuel Music \& the Moving Image organisé à l'Université de New York par les musicologues Gillian Anderson et Ronald Sadoff occupe une place déterminante dans l'institutionnalisation de la musicologie du cinéma outre-Atlantique: il s'agit du seul lieu d'échanges scientifiques régulier et international sur le sujet, qui réunit depuis 2005 une centaine de spécialistes issus de différentes disciplines, et s'est doté de son propre organe de publication du même nom. Anderson et Sadoff ont en outre annoncé, lors de la onzième édition en mai 2016, leur intention de fonder une Film Music Society - ce qui, à l'instar des Society for Music Analysis ou Society for Ethnomusicology par exemple, constituerait un pas important dans l'affirmation de la musicologie du cinéma comme une entité légitime à part entière.

La recherche française en musicologie du cinéma connaît un réel élan principalement depuis les années 2000 , avec la publication d'ouvrages collectifs et de monographies abordant des sujets variés, notamment autour de courants spécifiques, des cinéma nationaux ou des musiques de genre ${ }^{72}$. Ces études, qui s'appuient aussi bien sur les ouvrages de référence français qu'anglo-saxons, emblématisent l'effet d'entraînement suscité par les chercheurs anglophones souvent pris pour modèles dans les analyses. Depuis 2002, l'organisation de colloques centrés autour de la musique de cinéma joue également un rôle fondamental dans l'expansion de la recherche musicologique française ${ }^{73}$. En

70 Lerner 2010 ; Johnson 2010; Kalinak 2012; Halfyard 2012 ; Evans et Hayward 2016.

71 Voir Sadoff 2006, p. 165-83; Sadoff 2013, p. 663-81; Greene et Kulezic-Wilson 2016.

72 Masson et Mouëllic 2012; Rossi 2016 ; Carayol, Castanet et Pistone (dir.) à paraître en 2017.

73 Des colloques importants ont été organisés à l'Université Rennes 2 en mars 2002, à l'Institut catholique de Toulouse en décembre 2009, ou à l'Université de Rouen en mars 2015. Confirmant la perception nouvelle de la musique de film comme une branche disciplinaire légitime, la deuxième édition du colloque international "Recherches en musicologie: nouvelles perspectives» organisé à l'École Normale Supérieure de Lyon en septembre 2012 a accueilli une communication de Cécile Carayol sur l'esthétique musicale du cinéma français contemporain. 
pénétrant dans l'institution qu'est la Cité de la musique à l'occasion de l'exposition «Musique \& Cinéma, le mariage du siècle? » en 2013, la musique de film semble avoir conquis ses lettres de noblesse. Mentionnons enfin la constitution en France, fin 2015, du groupe de recherche international «Étude des Langages Musicaux à l'Écran», porté par un groupe de chercheurs francophones spécialistes de la musique et du son dans les médiums audiovisuels, dont Cécile Carayol (Université de Rouen), Jérôme Rossi (Université de Nantes), Emmanuelle Bobée (Université de Rouen), Hubert Bolduc-Cloutier (Université libre de Bruxelles/Université de Montréal), Chloé Huvet (Rennes 2/UdeM) et Jérémy Michot, (Rennes 2)74.

\subsection{Les organes de publication}

Cette ouverture ne peut que faire regretter l'absence d'organes de diffusion de la recherche française en musicologie du cinéma. Des disparités se dessinent là encore entre la France et les États-Unis. Il n'existe pas de revue scientifique francophone spécifiquement dédiée à la musique de film ${ }^{75}$, alors que des périodiques spécialisés en langue anglaise ont vu le jour, tels le Journal of Film Music (2002), Music, Sound and the Moving Image (2007), Music \& the Moving Image (2008) ou The New Soundtrack (2011). Surtout, plusieurs revues de musicologie réputées à l'étranger accueillent favorablement tous types de publications sur la musique à l'écran: le Journal of the Royal Musical Association au Royaume$\mathrm{Uni}^{76}$, 19th-Century Music, The Musical Quarterly, le Journal of the American Musicological Society ou Music Theory Online aux États-Unis ${ }^{77}$, Intersections, La Revue musicale OICRM ou Les Cahiers de la SQRM au Canada ${ }^{78}$. Un numéro récent de la revue Circuit: musiques contemporaines, intitulé "Musiques aux limites de l'image / Images at the Limits of Music», y est d'ailleurs intégralement consacré.

En France, force est de constater le manque d'espace accueillant des contributions sur la musique de film dans les revues savantes qui font autorité en musicologie, ce qui a conduit à une migration des publications vers les périodiques cinématographiques tels Simulacres ou Décadrages 79 . On peut toutefois relever l'article récent de Brice Tissier sur la Symphonie mécanique dans la Revue de Musicologie ${ }^{80}$. Le texte de Philippe Cathé sur la musique et les effets sonores de La Menace fantôme (1999) mérite également d'être signalé: certes, il reste

74 Après la journée d'étude «Reconfiguration des pratiques musicales dans le cinéma hollywoodien contemporain» organisée en novembre 2015 à Rennes 2 par Chloé Huvet et Jérémy Michot sous la supervision de Gilles Mouëllic, et celle organisée par Cécile Carayol à l'Université de Rouen en juin 2016 sur le thème "Analyses et esthétiques des duos réalisateurs-compositeurs", ce groupe a proposé un panel sur le timbre orchestral dans la musique de cinéma française et américaine au vingtième congrès de l'International Musicological Society à Tokyo en mars 2017.

75 À l'inverse, l'étude des musiques populaires par exemple s'est dotée en 2002 de la revue Volume!, accueillant à chaque numéro des contributions de musicologues et de spécialistes issus d'autres disciplines.

76 Voir Brown 2001, p. 275-303; Rogers 2004, p. 77-99.

77 Voir Citron 2011, p. 316-40; Lerner 2001, p. 477-515; Sheppard 2001, p. 303-357; Lehman 2013.

78 Voir Bowman 2006, p. 54-74; Hellégouarch 2015; Perron-Brault 2014, p. 9-21.

79 Voir par exemple Astic et Langlois 2002, p. 122-33; Vignon 2011, p. 30-47.

80 Tissier 2015, p. 367-406. 
l'unique publication dédiée à la musique de film dans Musurgia, mais les propositions de méthodologies d'analyse du continuum sonore signalent peut-être l'ébauche d'un arrimage ferme à la discipline, en fournissant aux musicologues un modèle pour l'analyse audio-visuelle tout en exploitant des outils fréquemment utilisés dans l'étude des musiques électroacoustiques ${ }^{81}$. Cet examen serait en effet incomplet sans l'exposé des problématiques que soulève l'étude musicologique de la musique de film, et la manière dont celles-ci questionnent la discipline dans ses pratiques et ses méthodes.

\section{MUSIQUE DE FILM ET MUSICOLOGIE : DÉFIS MÉTHODOLOGIQUES}

\subsection{Les sources}

Une première difficulté de taille se pose au musicologue: trouver des sources sur lesquelles travailler peut relever d'un parcours du combattant. Les partitions manuscrites sont éparpillées et majoritairement hors d'atteinte pour les chercheurs, ce qui pose des problèmes évidents tant pour l'analyse musicologique que pour une approche historique, qui sont des fondements majeurs de la discipline. Si la musique notée n’apparaît pas forcément nécessaire à l'appréhension de la musique de cinéma, comme le montrent par exemple les méthodes de Philip Tagg ${ }^{82}$ faisant peu appel à la musique écrite pour traiter les musiques de film, elle n'en reste pas moins un support majeur à l'analyse qui reste employé par une large majorité de musicologues du cinéma, anglophones comme francophones ${ }^{83}$.

L'ouvrage de Jeannie Pool et Stephen Wright, recensant tous les types de ressources disponibles pour l'étude de la musique du cinéma muet, les collections et fonds d'archive aux États-Unis par ordre alphabétique, constitue un outil très précieux pour les chercheurs s'intéressant aux productions américaines d'avant $1990^{84}$. Peu de partitions d'orchestre sont éditées ${ }^{85}$, et ce support même pose problème: rarement de la main du compositeur, ces partitions prennent la forme de recueils de pièces choisies n'excédant pas cinq cues. Dans le sillon de suites orchestrales tirées des productions scéniques savantes, ces partitions sont destinées à être interprétées en concert et correspondent rarement à la musique entendue dans le film. Elles sont ainsi de peu d'intérêt pour l'analyse audio-visuelle, même si, devant la pénurie de partitions, elles ne peuvent être totalement ignorées.

L'étude de la correspondance du compositeur et des sources primaires regroupant la correspondance, les contrats, le compte-rendu des spotting sessions, les mémos du monteur musique, les cue sheets et les notes prises pendant l'enregistrement, le mixage et le montage, n'est pas davantage envisageable dans la

81 Cathé 2007, p. 53-69. Nous avons volontairement exclu l'article de Roxane Hamery qui porte non pas sur la musique de film, mais sur la manière dont les Arabesques de Debussy ont trouvé une résonance particulière dans le cinéma d'avant-garde (Hamery 2010, p. 71-84).

82 Voir notamment Tagg et Clarida 2003.

83 Voir en particulier Heine 2016 ; et les articles publiés par Audrée Descheneaux, Jérôme Rossi et Cécile Carayol dans Toudoire-Surlapierre et Lécroart 2015, p. 135-85.

84 Pool et Wright 2011, p. 103-38.

85 L'éditeur Hal Leonard s'est cependant spécialisé dans la publication de partitions d'orchestre. 
majorité des cas (hors fonds d'archives). Ces archives sont la propriété des studios, qui refusent la plupart du temps toute collaboration avec les chercheurs dans la mesure où des enjeux financiers importants interviennent - situation redoublée dans le cas des franchises lucratives hollywoodiennes. Les documents sont classés et conservés en plusieurs exemplaires chez le compositeur, ses orchestrateurs, des éditeurs et des sociétés comme JoAnn Kane Music ${ }^{86}$. Celle-ci, qui gère notamment la bibliothèque musicale du studio Twentieth Century Fox, du département animation de DreamWorks, les catalogues Jerry Goldsmith et Alexandre Desplat, n'autorise pas davantage les chercheurs à accéder à ses collections: les partitions sont destinées à être louées à des orchestres qui souhaitent les interpréter en concert ${ }^{87}$. Très peu de donations sont par ailleurs faites à des bibliothèques ou des universités par des compositeurs contemporains, à l'exception notable de James Newton Howard, dont le geste de rendre accessibles ses partitions jusqu'aux plus récentes est en parfaite cohérence avec son projet pédagogique plus large de démocratiser la musique de film ${ }^{88}$. On rejoint là les problèmes qui se posent à toute étude musicologique contemporaine de son objet d'étude ${ }^{89}$.

Il est néanmoins possible de contourner ces difficultés. Les partitions de compositeurs savants ayant un temps travaillé pour le cinéma, comme Charles Koechlin ou Jacques Ibert, et celles de musiciens de film issus de la tradition savante tel Joseph Kosma, sont plus facilement accessibles et permettent de mener des analyses précises des manuscrits annotés ${ }^{\circ}$. Plusieurs jeunes chercheurs en musicologie se tournent aussi vers des archives privées qui comportent de nombreux documents inédits et encore peu exploités ${ }^{91}$. Les fonds d'archives des réalisateurs appellent également un dépouillement minutieux car de précieux documents relatifs à la musique y sont souvent conservés, comme c'est le cas dans le fonds Jean Grémillon à la Bibliothèque nationale de France ou François Truffaut à la Cinémathèque française ${ }^{92}$.

86 Les Éditions Mario Bois, par exemple, possèdent plusieurs partitions de Georges Delerue, notamment La nuit américaine (1973), Le retour de l'étalon noir (1983), Platoon (1986), ou Chouans! (1988).

87 La vogue des ciné-concerts depuis une dizaine d'années, aux billets très coûteux, s'inscrit dans cette logique de rentabilité.

88 Newton Howard (1951-), ancien étudiant à l'Université de Californie du Sud, y a assuré plusieurs cours de composition, et donne régulièrement des master classes. Des esquisses autographes et des partitions complètes entièrement orchestrées, de 1987 à 2009, sont conservées dans le fonds d'archives James Newton Howard des Special Collections de l'USC.

89 Sur le cas des études génétiques, voir Donin 2010, p. 13-36.

90 Voir Rossi 2010, p. 449-73; Rossi 2015, p. 151-71; Deschesneaux, 2016, p. 53-74.

91 Solenn Hellégouarch a ainsi pu consulter plusieurs partitions de Maurice Blackburn dans la collection de Louise Cloutier (Hellégouarch 2015), tandis que Chloé Huvet a eu accès à des documents d'archive et des partitions manuscrites du Mépris et de Vivement dimanche! auprès de Colette Delerue (Huvet 2010).

92 Le défrichement et l'analyse des documents musicaux du fonds Grémillon sont au cœur des recherches menées par Hubert Bolduc-Cloutier (2014). L'exploitation de la correspondance entre Truffaut et Delerue [Truffaut 456B222], des notes de travail [Truffaut $334 \mathrm{~B} 181$ ], et du document «Minutage musique du film» [Truffaut 162Bo98], de Vivement Dimanche! nous a permis d'analyser non seulement la manière dont le compositeur met en application les demandes musicales du réalisateur, 
Rencontrer directement les compositeurs ou leurs orchestrateurs semble à première vue être la solution la plus opérante puisqu'elle permet un accès à des sources manuscrites de première main et la réalisation d'entretiens. La consultation ne résout pas tout cependant, car bien souvent les auteurs ne reçoivent pas l'autorisation de reproduire des exemples à partir des partitions ${ }^{93}$. Entrer en contact avec les créateurs n'est d'ailleurs pas chose aisée: les agents font fréquemment barrage, et la difficulté d'accès croît proportionnellement à la notoriété du musicien, notamment à Hollywood. Des compositeurs comme Rolfe Kent ou Philippe Rombi ont ainsi reçu favorablement les demandes d'entretien de Jérémy Michot ou Cécile Carayol, alors qu'il a été impossible pour cette dernière de rencontrer Alexandre Desplat dans le cadre de sa thèse ${ }^{94}$. Aux ÉtatsUnis, rencontrer les compositeurs les plus renommés nécessite d'avoir un pied dans l'industrie ${ }^{95}$ ou de poursuivre une ambition de diffusion journalistique, à la teneur souvent promotionnelle ${ }^{96}$.

En l'absence de partitions, les transcriptions personnelles (mélodiques, rythmiques, harmoniques et/ou orchestrales) sont réalisées à l'écoute de la bande originale ou du film, soulevant plusieurs problèmes d'une autre nature. Les disques constituent un support appréciable bien que lacunaire: plusieurs cues sont absents, la bande originale n'en regroupant qu'une dizaine, tandis que d'autres y figurent alors qu'ils ne sont pas présents au montage ${ }^{97}$. Les pistes correspondent rarement à ce qui est entendu dans le film et nécessitent plusieurs précautions. Les exemples ci-dessous emblématisent chacun un cas de figure différent.

- L'ordre des pistes ne suit généralement pas la structure du film: des choix sont faits pour offrir une expérience d'écoute optimale, qui ne répond pas à la même logique que la narration cinématographique. Le cue «Cancelling the Apocalypse» composé par Ramin Djawadi pour Pacific Rim (2013) figure ainsi sur la plage 3 alors qu'il intervient seulement au dernier tiers du film.

- Les noms des pistes sont plus évocateurs qu'exacts; «Shawshank Redemption» ne donne qu'une idée vague de la situation réelle du cue de Thomas Newman dans Les Évadés (1994).

mais aussi d'élucider le recours à des types d'écriture qui paraissent étonnants dans sa production, voire contradictoires vis-à-vis de ses revendications esthétiques (Huvet 2016, p. 133-60).

93 Alors qu'il consacre un travail musicologique entier à la partition de The Dark Knight (2008), Vasco Hexel avertit en introduction: «Puisque, inexplicablement, les détenteurs des droits nous ont refusé l'autorisation de montrer des exemples musicaux détaillés, l'analyse de la partition repose ici en grande partie sur le système de notation de Helmholtz et des descriptions.» (Hexel 2016, p. xxi: «Since, inexplicably, the rightsholders denied permission to show detailed music examples, the score analysis here largely relies on Helmholtz pitch nomenclature and descriptions»).

94 Voir Michot 2015, p. 237-54; Carayol 2012.

95 C'est le cas de Ronald Sadoff, qui est aussi compositeur, producteur et superviseur musique. Il a pu avoir accès à des temp tracks, qui sont habituellement un point aveugle de la musicologie du cinéma. Voir Sadoff 2006, p. 165-83.

96 Voir par exemple Aschieri 1999.

97 C'est le cas par exemple du cue «Pleasured Distractions» composé par Trevor Morris pour la saison 1 des Tudors, présent sur la plage 3 du disque alors qu'il n'est jamais utilisé dans la série (2007-2010). 
- Les titres des pistes peuvent engendrer des confusions par rapport aux noms originels des cues. Sur le disque Le Mépris et autres films (2001), la plage "Ouverture» correspond au manuscrit «Capri» de Delerue, "Capri» au « $\mathrm{N}^{\circ} 8$ ", «Paul» au " $\mathrm{N}^{\circ} 5$ Chez Prokosch», et "La rupture chez Prokoch» au « $\mathrm{N}^{\circ} 10$ ».

- Les titres choisis sont parfois contradictoires avec l'action qu'ils accompagnent dans le film. Sur la bande originale de La Guerre des mondes (2005), le cue étrangement intitulé «The Separation of the Family» accompagne en fait l'arrivée de Ray et Rachel à Boston, où ils retrouvent Mary-Ann et Robbie.

- Un nécessaire retour au film doit donc être effectué, mais, si l'on s'intéresse avec précision aux choix compositionnels et à l'articulation entre la musique, les autres matériaux sonores et l'image, le mixage ne facilite pas toujours la perception distincte de chacun des éléments sonores, compliquant par ailleurs la transcription de l'accompagnement musical. La vitesse de lecture de la bande-son sur VHS ou DVD entraîne une transposition environ un demiton au-dessus de la hauteur originelle, dont il faut également tenir compte. Or, si le sens et la spécificité d'une scène émergent bien évidemment de la rencontre et de l'interdépendance entre sons, musique et images, plusieurs analystes ont souligné la nécessité de décomposer individuellement chacune de ces matières d'expression pour mieux appréhender ensuite leurs interactions ${ }^{98}$.

\subsection{Supports de publication et place de l'exemple}

Ce contexte audio-visuel, qui fonde l'essence de la musique de film, pose d'autres questions fondamentales de méthodologie disciplinaire.

Premièrement, le format papier encore prédominant est-il le plus pertinent pour les musicologues du cinéma alors qu'il ne rend compte que d'une partie seulement de l'expérience spectatorielle? Dans les analyses de séquence, les indications précises de minutage sont souvent absentes, et peu de chercheurs incluent des photogrammes à leurs articles - en particulier dans le cas de films hollywoodiens - afin d'éviter tout problème de droits d'auteur. Cela n'aide guère le repérage du lecteur qui souhaiterait confronter les observations proposées à sa propre perception, et dans le cas de films peu connus ou d'études détaillées de partitions rejetées au montage final, peut même constituer un obstacle à la lecture 99 .

Nombreux sont désormais les mémoires et les thèses à inclure les séquences analysées sur DVD ou clé USB, mais ce procédé ne s'est pas généralisé aux supports habituels de publication - pour des raisons financières et de droits pour les éditeurs. Confrontés à ce problème d'illustration audio-visuelle, des musicologues tels Philippe Langlois, Miguel Mera, James Buhler et David

98 Voir Neumeyer et Buhler 2001, p. 16-38; Buhler 2001, p. 39-61; Villani 2010, p. 73.

99 C'est le cas par exemple de David Cooper et Ian Sapiro qui, dans leur article sur Mélodie pour un meurtre (1989), se focalisent sur un seul cue finalement supprimé (Cooper et Sapiro 2008, p. 17-32). 
Neumeyer l'ont en partie résolu en accompagnant leurs articles papier de compléments audiovisuels mis en ligne sur des sites web ou des blogs ${ }^{100}$.

La musicologie du cinéma interroge ainsi le mode de présentation des exemples dans la discipline, réflexion développée dans le domaine des musiques du monde et des musiques populaires, mais aussi récemment autour des possibilités nouvelles déployées par les outils informatiques pour des types d'analyse déjà bien implantés ${ }^{101}$, et par les revues électroniques comme Musimédiane ou DÉMéter. Ces supports s'avèrent particulièrement intéressants non seulement pour des pratiques musicales qui s'accommodent mal du format papier sans de longues descriptions (comme l'étude de l'improvisation, de l'interprétation et les analyses acoustiques) ${ }^{102}$, mais aussi plus globalement, pour la pratique de l'analyse dans son entier, qui s'ouvrirait à une plus grande interactivité103. Plusieurs musicologues se sont ainsi tournés vers des supports de publication électroniques qui, en leur permettant d'inclure des extraits audio et vidéo en accompagnement de transcriptions sur portées ou de sonagrammes annotés pour étayer leurs analyses de film, offrent une précision, une clarté et un didactisme accrus ${ }^{104}$.

La musicologie du cinéma invite deuxièmement à repenser la place de l'exemple dans le discours sur la musique: comme pour l'étude des productions scéniques ou des musiques populaires, que retenir, que doit-on sélectionner pour rendre compte du "complexe audio-musico-visuel ${ }^{105}$ » sans être trop réducteur? Conscients de l'insuffisance du seul exemple sur portées, plusieurs chercheurs optent pour une présentation sous forme de tableau mettant en regard le matériau musical avec le minutage, une brève description de l'action, des dialogues et du montage ou les photogrammes correspondants ${ }^{106}$. Ces propositions sont loin d'être exhaustives et appellent certainement à être complétées dans le futur par d'autres formes de représentation; la meilleure manière de diffuser les recherches sur la musique de film demeure une question ouverte ${ }^{107}$.

On touche ainsi aux difficultés posées par l'interdisciplinarité qui reste peutêtre le défi majeur de la musicologie du cinéma. Les bases terminologiques et méthodologiques jetées par Gorbman et Chion, dont l'appui appréciable tant pour les chercheurs en cinéma qu'en musicologie a fait force de loi pendant

${ }^{100}$ Les sites http://www.lesclochesdatlantis.com/ et http://hearingthemovies.blogspot.ca/ accompagnent respectivement les ouvrages de Philippe Langlois, Les cloches d'Atlantis: musique électroacoustique et cinéma. Archéologie et histoire d'un art sonore (Paris: MF, 2012), et de James Buhler et David Neumeyer, Hearing the Movies (New York: Oxford University Press, 2016). Miguel Mera a publié sur son site personnel des extraits audio de plusieurs partitions qu'il analyse dans «Scoring Moth: Beyond the Temp Track», dans David Cooper, Christopher Fox et Ian Sapiro (éd.), CineMusic?, $33-50$.

101 Voir Goldman 2009, p. 107-22.

${ }^{102}$ Voir en particulier Canonne 2015; Chemillier 2010; Donin 2005.

103 Bossis 2005 .

104Voir Lehman 2013; Huvet 2015 et 2016.

105 Cardinal 2012, p. 47.

${ }^{106}$ Lehman 2015, p. 409-444; Deschesneaux 2016.

107 Une question similaire se pose pour la publication des partitions de musique de film et les choix éditoriaux qui doivent être faits. Plusieurs pistes sont proposées par Winters 2007, p. 115-140. 
plusieurs décennies, ont récemment été en partie réévaluées. Les problèmes soulevés par les choix terminologiques, les outils et les méthodes analytiques utilisés pour l'étude de la musique de film, rejoignant plus largement des préoccupations similaires en musicologie ${ }^{108}$, se voient en effet redoublés par l'évolution même des médiums audio-visuels. Le couple musique diégétique/ extra-diégétique, pour ne citer que cet exemple, apparaît trop rigide et inadéquat dans nombre de cas de figure ${ }^{109}$, et surtout pour les musiques utilisées dans les «vidéos virales», les sites internet et les jeux vidéo:

Un changement majeur est en train de s'opérer [...], et présumer que les modes de pensée développés par rapport à une forme narrative linéaire et fixée nous aident à comprendre l'audiovisualité dans une culture indéterminée, non linéaire et considérablement moins tournée vers la narration, paraît invraisemblable et malavisée ${ }^{110}$.

Outre ces problèmes relatifs au vocabulaire développé spécifiquement pour l'analyse audio-visuelle, la musique de film impose aux musicologues d'adopter une forme de décentrement et de développer des compétences doubles pour saisir avec acuité les rapports d'«entre-expression ${ }^{111}$ » entre le visuel et le sonore, interpréter leur rencontre, leur influence et leur enrichissement mutuels. Comment comprendre en effet les interactions entre musique et image sans connaître la grammaire du langage cinématographique ni tenir compte de la valeur expressive des échelles de plan choisies, des mouvements de caméra, du rythme du montage, du traitement de l'éclairage et des couleurs, du jeu d'acteur, de la gestion de l'espace, des décors ou des costumes?

Plusieurs auteurs issus tant du cinéma que de la musique, appellent ainsi à une prise en considération plus franche de la nature proprement intermédiatique de la musique de film sans renier pour autant les attaches à leur discipline mère ${ }^{112}$. Acquérir les méthodes et le vocabulaire d'un autre champ d'étude permet à la musicologie de «quitter son camp retranché ${ }^{113}$ » et de se nourrir en retour de compétences nouvelles, comme l'ont déjà montré les travaux aux méthodologies transdisciplinaires ${ }^{114}$. Il en va ainsi de la rencontre entre la chanson «Modern Love» de Bowie, l'entraînement irrépressible du corps, le «clignotement des lumières, des ombres et des couleurs ${ }^{115}$ » dans Mauvais Sang (1986), des reconfigurations audiovisuelles entre le matériau musical, la plastique de l'image et la caractérisation du personnage de Daniel Plainview

${ }^{108}$ Voir Donin 2009, p. 13-22; Kihm 2010, p. 21-38.

109Voir Altman 1987; Stilwell 2007, p. 184-202; Winters 2010, p. 224-44; Bobée 2011.

110 Kassabian 2013, p. 95: «A major shift is taking place [...], and to expect the thinking developed in relation to a determinate, linear narrative form to help us understand audiovisuality in an indeterminate, nonlinear, and substantially less narrative culture seems unlikely and unwise.»

111 Frangne 2003, p. 18.

112 Gorbman1998, p. 43; Villani 2010, p. 73.

113 Chimènes 1998, p. 73.

114 Voir Shephard et Leonard 2014. Toute la deuxième partie, constituée de neuf articles, est consacrée aux possibilités ouvertes par les méthodologies croisées entre l'histoire, la musicologie, les arts visuels et l'étude de l'interprétation (p. 25-74).

115 Cardinal 2012, p. 42. 
dans There Will Be Blood (2007) ${ }^{116}$, ou des juxtapositions déstabilisantes issues de la hantise d'un traumatisme passé qui infiltre les plans et la bande sonore de Katalin Varga (2009) ${ }^{117}$. Par ce type d'analyses entrecroisées, c'est la musicalité même du cinéma qui est mise au jour sous ses multiples facettes.

\section{RÉFÉRENCES}

Adorno, Theodor W. [1982] 2002. "On the Problem of Musical Analysis». Essays on Music, sous la dir. de Richard Leppert, 162-8o. Berkeley: University of California Press.

Adorno, Theodor W. et Hanns Eisler. [1944] 1972. Musique de cinema. Paris: L'Arche.

Altman, Rick. 1987. The American Film Musical. Bloomington: Indiana University Press.

—. 1992. «Four and a Half Film Fallacies». Sound Theory, Sound Practice, sous la dir. de Rick Altman, 35-45. Londres/New York: Routledge.

Aschieri, Roberto. 1999. Over The Moon: La Música de John Williams para el Cine. Santiago: Universidad Diego Portales.

Astic, Guy et Philippe Langlois. 2002. "La perception dans les plis: musique, son et silence dans Mulholland Drive». Simulacres, $\mathrm{n}^{\mathrm{0}} 6: 122-33$.

Barbedette, Sarah. 2014. Poétique du concert: à la lumière du tableau de Nicolas de Staël. Paris: Fayard.

Barnier, Martin. 2007. «Le cri de la nature chez Terrence Malick. Analyse du son de The Thin Red Line». Décadrages. Terrence Malick, $\mathrm{n}^{0} 11$ : 41-49.

2010. Bruits, cris, musiques de films. Les projections avant 1914. Rennes: Presses universitaires de Rennes.

Barthes, Roland. 1984. Le Bruissement de la langue. Paris : Éditions du Seuil. Becce, Giuseppe. 1920-1927. Kinothek: Neue Filmmusik. Berlin: Schlesinger.

Beck, Jay. 2013. "Acoustic Auteurs and Transnational Cinema». The Oxford Handbook of New Audiovisual Aesthetics, sous la dir. de John Richardson, Claudia Gorbman et Carol Vernallis, 732-751. New York: Oxford University Press.

Berthomieu, Pierre. 2011. Hollywood moderne: le temps des voyants. Pertuis: Rouge Profond.

Binns, Alexander. 2009. "The Development of Film Musicology: An Overview». Sound and Music in Film and Visual Media: An Overview, sous la dir. de Graeme Harper, Ruth Doughty et Jochen Eisentraut, 725-738. New York/Londres: Continuum.

Bobée, Emmanuelle. 2011. "Monde "réel" et monde imaginaire. Le rôle de la bande-son dans Eraserhead, de David Lynch ». Entrelacs, n ${ }^{\circ}$ 8. http://entrelacs.revues.org/236 (consulté le 2 mai 2015).

Bödeker, Hans E., Patrice Veit et Michael Werner (dir.). 2006. Organisateurs et organisations de concert en Europe, 1700-1920: institutionnalisation et pratiques. Berlin: Berliner Wissenschafts-Verlag. 
Boilès, Charles L. 1975. «La signification dans la musique de film ». Musique en jeu, $\mathrm{n}^{0}$ 19: 71-85.

Bolduc-Cloutier, Hubert. «La création musicale dans le cinéma des années trente en France: problématiques et mutations ». Thèse de doctorat débutée en 2014, Université libre de Bruxelles/Université de Montréal.

Bossis, Bruno. 2005. "Analyse en ligne: quelques réflexions». Musimédiane, $\mathrm{n}^{0}$ 1. http://www.musimediane.com/spip.php?article24 (consulté le 8 mai 2016).

Bowman, Durrell. 2006. «Dark Mirrors and Dead Ringers : Music for Suspense Films about Twins». Intersections:Canadian Journal of Music/Intersections: revue canadienne de musique $27, \mathrm{n}^{\circ} 1$ : 54-74.

Brown, Julie. 2001. "Ally McBeal's Postmodern Soundtrack». Journal of the Royal Musical Association 126, $\mathrm{n}^{\mathrm{O}} 2$ : 275-303.

Brown, Royal S. 1982. «Herrmann, Hitchcock, and the Music of the Irrational». Cinema Journal 21, $\mathrm{n}^{\circ} 2: 14-49$.

_.1994. Overtones and Undertones: Reading Film Music. Berkeley: University of California Press.

Bruce, Graham. 1985. Bernard Herrmann: Film and Narrative. Ann Arbor : UMI Research Press.

Buckland. 2006. Directed by Steven Spielberg: Poetics of the Contemporary Hollywood Blockbuster. New York: Continuum.

Buhler, James. 2001. "Analytical and Interpretative Approaches to Film Music (II): Analysing Interactions of Music and Film ». Film Music: Critical Approaches, sous la dir. de Kevin J. Donnelly, 39-61. Edinburgh: Edinburgh University Press.

Buhler, James et Alex Newton. 2013. "Outside the Law of Action: Music and Sound in the Bourne Trilogy». The Oxford Handbook of Sound and Image in Digital Media, sous la dir. de Carol Vernallis, Amy Herzog et John Richardson, 325-49. Oxford: Oxford University Press.

Buhler, James et David Neumeyer. 2016. Hearing the Movies. New York: Oxford University Press.

Burkholder, J. Peter. 1995. All Made of Tunes: Charles Ives and the Uses of Musical Borrowing. New Haven: Yale University Press.

Calabretto, Roberto. 2002. "Satie, Milhaud et le "collage musical" ». 1895. Mille huit cent quatre-vingt-quinze, $\mathrm{n}^{\mathrm{O}} 38$. https://1895.revues.org/225 (consulté le 2 mai 2015).

Canonne, Clément. 2015. «Le piano préparé comme "esprit élargi" de l'improvisateur: Une étude de cas autour du travail récent d'Ève Risser». Musimédiane, $\mathrm{n}^{\circ}$ 8. http://www.musimediane.com/spip.php?article178 (consulté le 9 mai 2016).

Carayol, Cécile. 2012. Une musique pour l'image: vers un symphonisme intimiste dans le cinéma français. Rennes: Presses universitaires de Rennes.

_ 2015. «La musique de film comme prolongement de l'opéra: correspondances entre The Wuthering Heights et The Ghost and Mrs Muir de Bernard Herrmann». Marges de l'opéra. Musique de scène, musique de 
film et musique radiophonique, 1920-1950, sous la dir. de Frédérique Toudoire-Surlapierre et Pascal Lécroart, 173-185. Paris: Vrin.

Carayol, Cécile et Jérôme Rossi (dir.). 2015. Musiques de séries télévisées. Rennes: Presses Universitaires de Rennes.

Carayol, Cécile, Pierre-Albert Castanet et Pascal Pistone (dir.). À paraître en 2017. Le fantastique dans les musiques des $X X^{e}$ et $X X I^{e}$ siècles. Sampzon: Éditions Delatour France.

Cardinal, Serge. 2012. "Où (en) est (l'étude de) la musique (au cinéma?) du film?». Intersections: Canadian Journal of Music/Intersections: revue canadienne de musique $33, \mathrm{n}^{\mathrm{0}} 1: 35-49$.

Cardinal, Serge, Martin Allard et Louis Comtois. 2002. «La musicalité d'une bande sonore. À propos de L'Invention d'un paysage». Écouter le cinéma, sous la dir. de Réal La Rochelle, 158-174. Montréal: Les 400 coups.

Carter, Erica (dir.). 2010. Béla Balázs: Early Film Theory. Visible Man and The Spirit of Film. New York/Oxford: Berghahn Books.

Cathé, Philippe. 2007. «Bruit et musique dans la course des Podracers de Star Wars, Episode I, The Fantome Menace (La Menace fantôme), 1999». Musurgia $14, \mathrm{n}^{0} 2: 53-69$.

Chanudaud, Stéphane. 2016. «La musique de film d'Arthur Honegger: du cinéma à l'écriture de concert». La musique de film en France: courants, spécificités, évolutions, sous la dir. de Jérôme Rossi, 35-52. Lyon: Symétrie. :

Chemillier, Marc. 2010. "De l'analyse acoustique à la modélisation des savoirs musicaux». Musimédiane, ${ }^{\circ}$ 5. http://www.musimediane.com/spip. php?article117 (consulté le 2 mai 2016).

Chimènes, Myriam.1998. "Musicologie et histoire. Frontière ou "no man's land" entre deux disciplines?». Revue de musicologie 84, $\mathrm{n}^{0}$ 1: 67-78.

Chion, Michel. 1990. L'audio-vision. Paris: Nathan.

-1995. La musique au cinéma. Paris: Fayard. 2010. Le son: traité d'acoulogie. Paris: Armand Colin.

Citron, Marcia J. 2008. «Poetics of Detachment: Tosca in the James Bond Film Quantum of Solace». 19th-Century Music 34, $\mathrm{n}^{\mathrm{O}} 3$ : 316-40.

Collins, Karen. 2008. Game Sound: An Introduction to the History, Theory, and Practice of Video Game Music and Sound Design. Cambridge: The MIT Press.

Cook, Nicholas. 1998. Analysing Musical Multimedia. Oxford: Clarendon Press. - [1998]. 2006. Musique, une très brève introduction, trad. de l'anglais par Nathalie Gentili. Paris: Éditions Allia.

Cooper, David. 2001. Bernard Herrmann's Vertigo: A Film Score Handbook. Westport: Greenwood Press.

-2005. Bernard Herrmann's The Ghost and Mrs. Muir: A Film Score Guide. Lanham: Scarecrow Press.

Cooper, David et Ian Sapiro. 2008. «Spotting, Scoring, Soundtrack: The Evolution of Trevor Jones's Score for Sea of Love». CineMusic?, Constructing the Film Score, sous la dir. de David Cooper, Christopher Fox et Ian Sapiro, 17-32. Newcastle : Cambridge Scholars Publishing.

Cugny, Laurent. 2009. Analyser le jazz. Paris: Éd. Outre mesure. 
Darby, William et Jack Du Bois.199o. American Film Music: Major Composers, Techniques, Trends, 1915-1990. Jefferson/Londres: McFarland Classics.

Daubney, Kate. 2000. Max Steiner's Now, Voyager: A Film Score Guide. Westport: Greenwood Press.

Deaville, James et Agnes Malkinson. 2014. "A Laugh a Second? Music and Sound in Comedy Trailers». Music, Sound and the Moving Image 8, $\mathrm{n}^{\mathrm{o}} 2$ : 121-40.

DeNora, Tia. 1998. Beethoven et la construction du génie: musique et société à Vienne, 1792-1803, trad. de l'anglais par Marc Vignal. Paris: Fayard.

Descheneaux, Audrée. 2010. "Unité et fonctionnalité de la musique de film chez Koechlin». Charles Koechlin: compositeur et humaniste, sous la dir. de Philippe Cathé, Sylvie Douche et Michel Duchesneau, 475-91. Paris: Vrin.

_. 2016. "Musique et idées dans La Grande Illusion (1937-1958)». La musique de film en France: courants, spécificités, évolutions, sous la dir. de Jérôme Rossi, 53-74. Lyon: Symétrie.

Donin, Nicolas. 2005. "Samson François jouant Noctuelles: notes de lecture», DÉMéter. http://demeter.revue.univ-lille3.fr/interpretation/donin/web/ doninweb.html (consulté le 4 mai 2016).

—. 2009. "Analyser l'analyse?». L'analyse musicale, une pratique et son histoire, sous la dir. de Rémy Campos et Nicolas Donin, 22-27. Genève: Droz.

—_. 2010. "Quand l'étude génétique est contemporaine du processus de création: nouveaux objets, nouveaux problèmes». Genesis, $\mathrm{n}^{0}$ 31:13-36.

Donnelly, Kevin J., William Gibbons et Neil Lerner (dir.). 2014. Music in Video Games: Studying Play. New York/Abingdon: Routledge.

Doucet, Jeanne. 2016. «Le timbre comme élément signifiant dans la musique de jeu vidéo: le cas de World of Warcraft». Mémoire de maîtrise, Université de Montréal.

Eaton, Rebecca M. 2008. «Unheard Minimalisms: The Functions of the Minimalist Techniques in Film Scores». Thèse de doctorat, The University of Texas at Austin.

Eugène, Jean-Pierre. 200o. Les Musiques dans les films d'Alfred Hitchcock. Paris: Dreamland.

Evans, Mark et Philip Hayward (dir.). 2016. Sounding Funny: Music, Sound and Comedy Cinema. Sheffield: Equinox.

Flinn, Caryl. 1992. Strains of Utopia: Gender, Nostalgia and Hollywood Film Music. Princeton: Princeton University Press.

Frangne, Pierre-Henry. 2003. "Introduction: musique et image au cinéma». Musiques et images au cinéma, sous la dir. de Marie-Noëlle Masson et Gilles Mouëllic, 17-25. Rennes: Presses universitaires de Rennes,

Gimello-Mesplomb, Frédéric. 2016. «Tendances et spécificités de la musique dans le cinéma de la Nouvelle Vague». La musique de film en France: courants, spécificités, évolutions, sous la dir.de Jérôme Rossi, 109-32. Lyon: Symétrie,.

Gloag, Kenneth. 2012. Postmodernism in Music. Cambridge: Cambridge University Press. 
Goldman, Jonathan. 2008. «De la contemporanéité de l'arrangement». Circuit: musiques contemporaines. Postiches et mélanges $18, \mathrm{n}^{\circ} 2: 8$.

_. 2009. "Un outil de "mise en tableau" au service de l'analyse paradigmatique, et quelques divergences interprétatives». L'analyse musicale, une pratique et son histoire, sous la dir. de Rémy Campos et Nicolas Donin, 107-22. Genève: Droz.

Gonin, Philippe. 2008. Tempus Perfectum. Aux sources de la musique de film: Le Film d'Art, L'Assassinat du duc de Guise et Camille Saint-Saëns, $\mathrm{n}^{\mathrm{O}} 5$.

Gorbman, Claudia.1987. Unheard Melodies: Narrative Film Music. Londres/ Bloomington: BFI Publishing/Indiana University Press.

-1995. «The State of Film Music Criticism». Cineaste 21, $\mathrm{n}^{0} 1-2$ : 72-75.

—.1998. «Film Music». The Oxford Guide to Film Studies, sous la dir. de John Hill et Pamela Church Gibson, 41-48. Londres: Oxford University Press.

Greene, Liz et Danijela Kulezic-Wilson (dir.). 2016. The Palgrave Handbook of Sound Design and Music in Screen Media. Basingstoke: Palgrave Macmillan.

Guido, Laurent. 2006. «Entre opéra wagnérien et culture de masse : l'univers musical de Star Wars». Décadrages, $\mathrm{n}^{\circ} 8-9: 52-75$.

Hacquard, Georges. 1959. La musique et le cinéma. Paris: Presses universitaires de France.

Halfyard, Janet K. 2013. "Cue the Big Theme? The Sound of the Superhero". The Oxford Handbook of New Audiovisual Aesthetics, sous la dir. de John Richardson, Claudia Gorbman et Carol Vernallis, 171-93. New York: Oxford University Press.

-2012. The Music of Fantasy Cinema. Sheffield: Equinox.

Hamery, Roxane. 2010. «Debussy tel qu'en lui-même le cinéma l'a vu (et entendu): Arabesques de Germaine Dulac et Images pour Debussy de Jean Mitry». Musurgia 17, $\mathrm{n}^{\mathrm{0}} 2:$ 71-84.

Hanslick, Eduard. [1854] 2012. Du Beau musical: contribution à la réforme de l'esthétique musicale, trad. de l'allemand par Alexandre Lissner. Paris: Herman.

Heine, Erik. 2016. James Newton Howard's Signs: A Film Score Guide. Lanham, Rowman \& Littlefield.

Hellégouarch, Solenn. 2015. «David Cronenberg et Howard Shore. Bref portrait d'une longue collaboration ». Revue musicale OICRM 2, $\mathrm{n}^{\circ}$ 2. http://revuemusicaleoicrm.org/rmo-vol2-n2/ (consulté le 9 août 2015).

_. 2015. "Une méthode dangereuse. Comprendre le processus créateur en musique de film: le cas de Norman McLaren et Maurice Blackburn, David Cronenberg et Howard Shore». Thèse de doctorat, Université de Montréal.

Hexel, Vasco. 2016. Hans Zimmer and James Newton Howard's The Dark Knight: A Film Score Guide. Lanham: Rowman \& Littlefield.

Hickman, Roger. 2011. Miklós Rózsa’s Ben-Hur: A Film Score Guide. Lanham: Scarecrow Press. 
Huvet, Chloé. 2010. "Georges Delerue: une esthétique musicale et sa mise en application dans deux films de Jean-Luc Godard et François Truffaut (1963-1983)». Mémoire de master 1, École Normale Supérieure de Lyon.

_- 2014. «La dissociation musique/images dans Jurassic Park: un élargissement des pratiques compositionnelles de l'âge d'or hollywoodien dans la partition de John Williams». Cahiers de la Société Québécoise de Recherche en Musique 15, $\mathrm{n}^{\circ} 2: 23-39$.

_. 2015. "The Tudors de Trevor Morris: l'importation du style "Media Ventures" dans une série télévisée?». Musiques de séries télévisées, sous la dir. de Cécile Carayol et Jérôme Rossi, 119-22. Rennes: Presses Universitaires de Rennes.

__ 2015. "Musique et effets sonores dans Star Wars: Episode II-L'Attaque des clones: une alliance conflictuelle?». Revue musicale OICRM $2, \mathrm{n}^{\mathrm{o}} 2$ : http://revuemusicaleoicrm.org/rmo-vol2-n2/musique-et-effets-sonoresdans-star-wars-episode-ii-lattaque-des-clones-une-alliance-conflictuelle$\% \mathrm{E} 2 \% 80 \% 89 /$ (consulté le 2 août 2015).

—. 2016. "Vivement Dimanche! de Georges Delerue (1983): un hommage distancié aux films classiques américains». La musique de film en France: courants, spécificités, évolutions, sous la dir. de Jérôme Rossi, 133-6o. Lyon: Symétrie.

- 2016. "Le Pianiste de Roman Polanski (2002). Survivre et exister par la musique». Revue musicale OICRM 3 «Mémoire musicale et résistance: autour du Verfügbar aux Enfers de Germaine Tillion», sous la direction de Marie-Hélène Benoit-Otis et Philippe Despoix, $\mathrm{n}^{0}$ 2. http://revuemusicaleoicrm.org/rmo-vol3-n2/le-pianiste/ (consulté le 7 juin 2016).

Jaubert, Maurice. 1936. «Les Arts. Le Cinéma. Petite école du spectateur (suite). La Musique». Esprit, no 43:114-19.

Johnson, Bruce (dir.). 2010. Earogenous Zones: Sound, Sexuality and Cinema. Londres: Equinox.

Julien, Jean-Rémy. 1980. «Éléments méthodologiques pour une typologie de la musique de film». Revue de musicologie 66, $\mathrm{n}^{\circ} 2: 179-202$.

Jullier, Laurent. 2009. «To Cut or Let Live: The Soundtrack According to JeanLuc Godard». Sound and Music in Film and Visual Media: An Overview, sous la dir. de Graeme Harper, Ruth Doughty et Jochen Eisentraut, 352-62. New York: Continuum.

_. 2011. L'analyse de sequences. Paris: Armand Colin.

Kalinak, Kathryn 1982. "The Fallen Woman and the Virtuous Wife: Musical Stereotypes in The Informer, Gone with the Wind, and Laura». Film Reader, $\mathrm{n}^{\mathrm{O}} 5: 76-82$.

—.1992. Settling The Score: Music and the Classical Hollywood Film. Madison: University of Wisconsin Press.

- 2007. How the West Was Sung: Music in the Westerns of John Ford. Berkeley: University of California Press.

__ 2010. Film Music: A Very Short Introduction. Oxford: Oxford University Press. 
(dir.) 2012. Music in the Western: Notes From the Frontier. New York/ Abingdon: Routledge.

Kaltenecker, Martin. 2010. L'Oreille divisée. Les discours sur l'écoute musicale aux xviiie et xixe siècles. Paris : Musica Falsa.

Kamp, Michiel, Tim Summers et Mark Sweeney (dir.). 2016. Ludomusicology: Approaches to Video Game Music. Sheffield/Bristol : Equinox Publishing.

Karlin, Fred et Rayburn Wright. [1990] 2004. On the Track: A Guide to Contemporary Film Scoring. New York: Routledge.

Kassabian, Anahid. 2001. Hearing Film: Tracking Identifications in Contemporary Hollywood Film Music. New York: Routledge.

- 2013. "The End of Diegesis as We Know It?». The Oxford Handbook of New Audiovisual Aesthetics, sous la dir. de John Richardson, Claudia Gorbman et Carol Vernallis, 89-106. New York: Oxford University Press.

Kerman, Joseph. 1983. "A Few Canonic Variations». Critical Inquiry 10, $\mathrm{n}^{\circ} 1$ : $107-25$.

Kickasola, Joseph G. 2012. "Kieślowski’s musique concrète». Music, Sound and Filmmakers: Sonic Style in Cinema, sous la dir. de James Wierzbicki, 61-75. New York: Routledge.

Kihm, Christophe. 2010. «Typologie de la reprise». Volume! 7, n ${ }^{\circ} 1: 21-38$.

Kracauer, Siegfried. 1960. Theory of Film: The Redemption of Physical Reality. Londres/Oxford/New York: Oxford University Press.

Kulezic-Wilson, Danijela. 2008. "Sound Design is the New Score». Music, Sound, and the Moving Image 2, $\mathrm{n}^{\mathrm{O}} 2: 127-31$.

- 2011. "Soundscapes of Trauma and the Silence of Revenge in Peter Strickland's Katalin Varga». The New Soundtrack 1, n ${ }^{0} 1: 57-71$.

Lacasse, Serge. 2006. "Composition, performance, phonographie: un malentendu ontologique en analyse musique?». GROOVE: enquête sur les phénomènes musicaux contemporains. Mélanges à la mémoire de Roger Chamberland, sous la dir. de Patrick Roy et Serge Lacasse, 65-78. Québec: Presses de l'Université Laval.

Lacombe, Alain et François Porcile. 1995. Les musiques du cinéma français. Paris: Bordas.

Lahire, Bernard. 2004. La culture des individus: dissonances culturelles et distinction de soi. Paris: La Découverte.

Langlois, Gérard.1968. «Georges Delerue, le Mélomane Cinéphile». Les Lettres Françaises, $\mathrm{n}^{\mathrm{O}} 1225$.

Langlois, Philippe. 2012. Les cloches d'Atlantis: musique électroacoustique et cinéma. Archéologie et histoire d'un art sonore. Paris: MF.

Larsen, Peter. [2005] 2007. Film Music. Londres : Reaktion Books.

Lehman, Frank. 2013. "Hollywood Cadences: Music and the Structure of Cinematic Expectation». MTO 19, $\mathrm{n}^{\circ} 4 \mathrm{http} / /$ www.mtosmt.org/issues/mto .13.19.4/mto.13.19.4.lehman.html (consulté le 7 février 2016).

Lehman, Frank. 2015. «Scoring the President: Myth and Politics in Williams's JFK and Nixon». Journal of the Society for American Music 9, $\mathrm{n}^{\circ} 4: 409-444$.

Lerner, Neil. 2001. "Copland's Music of Wide Open Spaces: Surveying the Pastoral Trope in Hollywood». The Musical Quarterly 85, $\mathrm{n}^{\mathrm{0}} 3: 477-515$. 
. (dir.). 2010. Music in the Horror Film: Listening to Fear. Londres : Routledge.

Levinson, Jerrold. 1990. «Musical Expressiveness». The Pleasures of Aesthetics, 90-125. Ithaca: Cornell University Press.

McDonald, Matthew. 2012. "Mountains, Music and Murder. Scoring the American West in There Will Be Blood and No Country for Old Men». Music in the Western: Notes from the Frontier, sous la dir. de Kathryn Kalinak, 214-27. New York: Routledge.

Machart, Renaud. 1995. Poulenc. Paris: Seuil.

Marks, Martin. 1982. «Film Music: The Material, Literature and Present State of Research ». Journal of the University Film and Video Association $34, \mathrm{n}^{\circ} 1$ : 3-40.

-1997. Music and the Silent Film: Contexts and Case Studies, 1895-1924. New York : Oxford University Press.

Martin, Roxane. 2007. La féérie romantique sur les scènes parisiennes (17911864). Paris: Champion.

Masson, Marie-Noëlle et Gilles Mouëllic (dir.). 2003. Musiques et images au cinéma. Rennes: Presses universitaires de Rennes.

McGill, Amy C. 2008. «The Contemporary Hollywood Film Soundtrack: Professional Practices and Sonic Styles Since the 1970s». Thèse de doctorat, Université d'Exeter.

Mera, Miguel. 2008. «Scoring Moth: Beyond the Temp Track». CineMusic? Constructing the Film Score, sous la dir. De David Cooper, Christopher Fox et Ian Sapiro, 33-50. Newcastle : Cambridge Scholars Publishing.

Michot, Jérémy. 2015. "Analyse comparative des représentations musicales de la mort dans les génériques de séries télévisées (Six Feet Under, Dexter, The Walking Dead et Les Revenants)». Musiques de séries télévisées, sous la dir. de Cécile Carayol et Jérôme Rossi, 237-54. Rennes: Presses Universitaires de Rennes.

—_. "Mise en série et intertextualité: les enjeux musicaux de la série télévisée», Thèse de doctorat débutée en 2012, Université Rennes 2.

Minett, Mark. 2013. «Beyond the Badass: Electronic Danse Music Meets Film Music Practice». New Review of Film and Television Studies 11, $\mathrm{n}^{0} 2: 191-210$. Mouëllic, Gilles. 200o. Jazz et cinéma. Paris: Cahiers du cinéma. . 2003. La musique de film. Paris: Cahiers du Cinéma/SCÉRÉN-CNDP.

Nattiez, Jean-Jacques (éd.). 2003-2007. Musiques: Une encyclopédie pour le $X X I^{e}$ siècle Arles/Paris: Actes Sud/Cité de la musique, 5 volumes.

Neumeyer, David et James Buhler. 2001. "Analytical and Interpretative Approaches to Film Music (I): Analysing the Music». Film Music: Critical Approaches, sous la dir. de Kevin J. Donnelly, 16-38. Edinburgh: Edinburgh University Press.

Neumeyer, David et Nathan Platte. 2011. Franz Waxman's Rebecca: A Film Score Guide. Lanham: Scarecrow Press.

Oddos, Philippe et Christian Carcassonne. 1979. «Entretien avec Pierre Boulez». Cinématographe, $\mathrm{n}^{0} 52: 25-27$. 
Orosz, Jeremy. 2015. «John Williams: Paraphraser or Plagiarist?». Journal of Musicological Research 34, $\mathrm{n}^{0} 4: 299-319$.

Perron-Brault, Alexis. 2014. «De l'ONF à Télé-Québec: le parcours de Pierre F. Brault, compositeur de musique de film». Cahiers de la Société Québécoise de Recherche en Musique 15, $\mathrm{n}^{\mathrm{0}} 2$ : 9-21.

Peterson, Richard. 1992. «Understanding Audience Segmentation: From Elite and Mass to Omnivore and Univore». Poetics $21: 243-58$.

Pisano, Giusy. 2002. «Sur la présence de la musique dans le cinéma dit muet». 1895. Mille huit cent quatre-vingt-quinze, $\mathrm{n}^{\circ} 38 \mathrm{http}: / / 1895$.revues.org/218 (consulté le 2 mai 2015).

Pistone, Pascal. 2006. «Musicologie et cinéma. État des lieux, perspectives de recherche et bibliographie». Musicologie et cinéma, sous la dir. de Pascal Pistone, 89-129. Paris: Université de Paris-Sorbonne.

Poirier, Alain. 2003. "Les fonctions de la musique au cinéma». Musiques: Une encyclopédie pour le XXI siècle, "1. Musiques du XX $\mathrm{XX}^{\mathrm{e}}$ siècle», sous la dir. de Jean-Jacques Nattiez, 750-55. Arles/Paris: Actes Sud/Cité de la musique.

Pool, Jeannie G. et H. Stephen Wright. 2011. A Research Guide to Film and Television Music in the United States. Lanham: Scarecrow Press.

Porcile, François. 1971. Maurice Jaubert: musicien populaire ou maudit? Paris: Les Éditeurs français réunis.

Ramaut-Chevassus, Béatrice. 1998. Musique et postmodernité. Paris: Presses universitaires de France.

Rapée, Ernö. 1925. Encyclopedia of Music for Pictures. New York: Belwin.

Rebichon, Michel.1987. «Georges Delerue Fortissimo». Studio, $\mathrm{n}^{\circ} 2$ : 94-99.

Riesman, Michael. 2016. "Foreword». Shared Meanings in the Film Music of Philip Glass: Music, Multimedia and Postminimalism, sous la dir. de Tristian Evans, xiii. Abingdon/New York: Routledge.

Reibel, Emmanuel. 2015. "Réflexions sur l'opéra français au tournant du XXI siècle». La création lyrique en France depuis 1900: contexte, livrets, marges, sou la dir. de Cécile Auzolle, 73-84. Rennes : Presses Universitaires de Rennes.

Rodgers, Stephen. 2009. Form, Program, and Metaphor in the Music of Berlioz. New York: Cambridge University Press.

Rodman, Ronald. 2000. "Tonal Design and the Aesthetic of Pastiche in Herbert Stothart's Maytime». Music and Cinema, sous la dir. de James Buhler, Caryl Flynn et David Neumeyer, 187-206. Hanover: Wesleyan University Press.

Rogers, Holly. 2004. «Fitzcarraldo’s Search for Aguirre: Music and Text in the Amazonian Films of Werner Herzog ». Journal of the Royal Musical Association $129, \mathrm{n}^{0} 1: 77-99$.

Rosar, William H. 2003. «Bernard Herrmann: The Beethoven of Film Music? ». The Journal of Film Music 1, $\mathrm{n}^{\mathrm{O}}$ 2-3: 121-50.

Rosar, William H. 2009. «Film Studies in Musicology: Disciplinarity vs. Interdisciplinarity». The Journal of Film Music 2, $\mathrm{n}^{0}$ 2-4: 99-125.

Rossi, Jérôme. 2010. «Koechlin et la musique pour l'image: Victoire de la vie de Henri Cartier-Bresson ». Charles Koechlin: compositeur et humaniste, sous 
la dir. de Philippe Cathé, Sylvie Douche et Michel Duchesneau, 449-73. Paris: Vrin.

.2011. «Le dynamisme harmonique dans l'écriture filmique de John Wil-

liams: harmonie fonctionnelle versus harmonie non fonctionnelle». John

Williams: un alchimiste musical à Hollywood, sous la dir. de Alexandre Tylski, 113-40. Paris: L'Harmattan.

- 2015. "De la musique de scène à la musique de film: Macbeth d'Orson Welles et Jacques Ibert, une écriture musico-cinématographique». Marges de l'opéra. Musique de scène, musique de film et musique radiophonique, 1920-1950, sous la dir. de Frédérique Toudoire-Surlapierre et Pascal Lécroart, 151-71. Paris: Vrin.

(dir.). 2016. "Introduction». La musique de film en France: courants, spécificités, évolutions, 7-31. Lyon: Symétrie.

- 2016. La musique de film en France: courants, spécificités, évolutions. Lyon: Symétrie.

Roustom, Kareem. 2014 "Michel Legrand Scores Une femme est une femme». A Companion to Jean-Luc Godard, sous la dir. de Tom Conley et T. Jefferson Kline, 71-88. Chichester: Wiley Blackwell.

Sadoff, Ronald H. 2006. "The Role of the Music Editor and the "Temp Track" as Blueprint for the Score, Source Music, and Scource Music of Films ». Popular Music 25, $\mathrm{n}^{\mathrm{O}} 2: 165-83$.

_. 2013. «Scoring for Film and Video Games: Collaborative Practices and Digital Post-production »,). The Oxford Handbook of Sound and Image in Digital Media, sous la dir.de Carol Vernallis, Amy Herzog et John Richardson, 663-81. Oxford: Oxford University Press.

Shephard, Tim et Anne Leonard (dir.). 2014. The Routledge Companion to Music and Visual Culture. New York/Abingdon: Routledge.

Sheppard, W. Anthony 2001. "An Exotic Enemy: Anti-Japanese Musical Propaganda in World War II Hollywood». Journal of the American Musicological Society $54, \mathrm{n}^{\mathrm{O}} 2: 303-57$.

Smith, Steven C. 2002. A Heart at Fire's Center: The Life and Music of Bernard Herrmann. Berkeley: University of California Press.

Soury, Thomas. 2013. «Louis de Cahusac, librettiste et théoricien: un collaborateur majeur à l'œuvre de Rameau ». Revue de musicologie 99, n 1 : 33-60.

Stilwell, Robynn J. 2002. «Music in Films: A Critical Review of Literature, 1980-1996». The Journal of Film Music 1, $\mathrm{n}^{0} 1: 19-61$.

- 2007. "The Fantastical Gap between Diegetic and Non-Diegetic». Beyond the Soundtrack. Representing Music in Cinema, sous la dir. de Daniel Goldmark, Lawrence Kramer et Richard Leppert, 184-202. Berkeley: University of California Press.

—_. 2011. " "Bad Wolf": Leitmotif in Doctor Who (2005)». Music in Television: Channels of Listening, sous la dir. de James Deaville, 119-42. New York/Abingdon: Routledge.

Stravinsky, Igor. 1947. «La Musique de film? Du papier peint!». Écran français $\mathrm{n}^{\mathrm{0}} 125: 3$. 
Tagg, Philip et Bob Clarida. 2003. Ten Little Title Tunes: Towards a Musicology of the Mass Media. New York/Montréal: Mass Media Music Scholars' Press.

Terrien, Pascal (dir.). 2014. Musique française. Esthétique et identité en mutation 1892-1992 Sampzon: Éditions Delatour France.

Tissier, Brice. 2015. «Symphonie mécanique de Pierre Boulez: étude d'une dérive cinématographique». Revue de musicologie 101, $\mathrm{n}^{0} 2$ : 367-406.

Vignon, Gaspard. 2011. «Ludwig van Beethoven - Gus Van Sant: vers un idéal romantique. Musique et silence dans Elephant». Décadrages. Autour d'Elephant de Gus Van Sant, $\mathrm{n}^{\circ}$ 19:30-47.

Villani, Vivien. 2010. "Analyser les musiques de film: quelques études de cas». Analyser la musique de film: méthodes, pratiques, pédagogie, sous la dir. de Frédéric Gimello-Mesplomb, 76-88. Norderstedt: Books on Demand.

Webster, Jamie L. . 2009. "The Music of Harry Potter: Continuity and Change in the First Five Films». Thèse de doctorat, University of Oregon.

Winters, Ben. 2007. Erich Wolfgang Korngold's The Adventures of Robin Hood: A Film Score Guide. Lanham: Scarecrow Press.

- 2007. «Catching Dreams: Editing Film Scores for Publication». Journal of the Royal Musical Association 132, $\mathrm{n}^{\circ} 1: 115-140$.

-2010. "The Non-Diegetic Fallacy: Film, Music, and Narrative Space». Music \& Letters 91, n 2 : 224-44.

Wright, Benjamin. 2015. «Music and the Moving Image: A Case Study of Hans Zimmer». The Routledge Reader on the Sociology of Music, sous la dir. de John Shepherd et Kyle Devine, 319-27. New York: Routledge.

Zwein-Chouard, Cédrine. 2016. "Yves Baudrier: le cinéma comme nouveau "terrain d'entente" de la musique française ». La musique de film en France: courants, spécificités, évolutions, sous la dir. de Jérôme Rossi, 97-107. Lyon: Symétrie.

\section{RÉSUMÉ}

Si la musique de cinéma a longtemps été délaissée sur le plan scientifique, en particulier dans la francophonie, la floraison d'analyses musicologiques spécialisées dans ce domaine depuis quelques décennies commence toutefois à combler cette lacune. Par un regard réflexif porté sur ce jeune champ disciplinaire qu'est la «musicologie du cinéma», il s'agira d'interroger la place de l'étude de la musique de film au sein des travaux en musicologie, afin de comprendre le désaveu dont elle a pu faire l'objet. Bien que des disparités perdurent entre les musicologies nord-américaine et française, nous montrerons que l'institutionnalisation progressive de l'étude de la musique de film et son rattachement assumé à la musicologie semblent en bon chemin. L'exposé des problématiques propres à l'étude de la musique de cinéma permettra de dégager non seulement les défis méthodologiques posé par un tel objet de recherche, mais aussi d'éclairer la manière dont elles rejoignent et réengagent nombre de réflexions animant la discipline musicologique elle-même. 


\begin{abstract}
Film music has been neglected for a long time in scholarly literature, especially in French-speaking countries. This shortcoming is addressed since a few decades by the blossoming of musicological and specialized analysis devoted to film music. By casting a retrospective glance over this young academic field called "film musicology", we will first question the place of film music study within musicological research, so as to understand the disavowal it has been subjected to. Disparities still exist between North American and French approaches, but we will demonstrate that the progressive institutionalization of film music and its inclusion within musicology are set on an encouraging path. The issues specific to film music study will be examined in order to highlight what methodological challenges such a research object entails. We will also show how they relate to and reengage a number of reflections taking place within musicology itself.
\end{abstract}

\title{
BIOGRAPHIE
}

Chloé Huvet est docteure de l'Université Rennes 2 et de l'Université de Montréal, où sa thèse sur la musique et le sound design des deux trilogies de la saga Star Wars sous la direction de Gilles Mouëllic et Michel Duchesneau a reçu la mention «Exceptionnel». Ancienne élève de l'École Normale Supérieure de Lyon, elle est Major de l'agrégation externe de musique en 2012 et a participé à de nombreux colloques internationaux sur la musique à l'image. Ses publications comprennent notamment des articles sur la musique d'Eyes Wide Shut (S. Kubrick, 1999), de Vivement dimanche! (F. Truffaut, 1993), de Jurassic Park (S. Spielberg, 1993), du Pianiste (R. Polanski, 1999), de la série télévisée Les Tudors (2007), sur la musique, le sound design et les technologies numériques dans Star Wars, et sur les compositeurs Wojciech Kilar et Bruno Coulais. Chargée de cours en histoire et analyse de la musique de cinéma depuis 2014 à l'Université de Montréal, elle est adjointe à la direction générale de la Société de musique contemporaine du Québec en 2016-2017. Elle est la nouvelle rédactrice en chef de L'Opéra - Revue québécoise d'art lyrique, et la coordinatrice éditoriale de la Revue musicale OICRM, où elle dirige un numéro sur la bande-son des blockbusters contemporains, à paraître fin 2018. 